Research Article

\title{
Large Scale Gas Stratification Erosion by a Vertical Helium-Air Jet
}

\author{
R. Kapulla, G. Mignot, S. Paranjape, L. Ryan, and D. Paladino
}

Paul Scherrer Institut, 5232 Villigen, Switzerland

Correspondence should be addressed to R. Kapulla; ralf.kapulla@psi.ch

Received 5 August 2014; Accepted 25 September 2014; Published 30 November 2014

Academic Editor: Arkady Serikov

Copyright (C) 2014 R. Kapulla et al. This is an open access article distributed under the Creative Commons Attribution License, which permits unrestricted use, distribution, and reproduction in any medium, provided the original work is properly cited.

Containment conditions after certain postulated severe accident scenarios in nuclear power plants might result in the accumulation of hydrogen in the vessel dome. Inspired by these accident scenarios an experiment for the OECD/NEA benchmark exercise (2014) was carried out in the large scale PANDA facility at the Paul Scherrer Institut in Switzerland. The benchmark experiment was conducted at room temperature and under conditions characterized by an initially positively buoyant jet which becomes negatively buoyant while interacting with a helium layer. The experiment addresses (i) the initial conditions especially at the tube exit and (ii) the details of the entrainment of the helium stratification into the jet and the transport of the mixture towards the lower parts of the vessel. For the tube exit velocity mean and fluctuating quantities we find a reasonable agreement with pipe flow data, but a lack of agreement between past tube exit measurements and our results. It is shown that the axial velocity of the jet experiences a strong deceleration in the vicinity of the helium-rich layer and is finally stopped. Fluid accumulates in this zone and part of this fluid is flowing back in a narrow annular region around the upward flowing jet. Consequently, part of the annular flow is reentrained into the rising jet. During the layer erosion, the flow structure changes from a more downwards oriented annular type to a more horizontally oriented mushroom type of flow. It is found that locations for which we record considerable turbulent kinetic energy $k$ extends above the region where the velocity magnitude $|v|$ has decayed to almost zero, indicating that the jet deceleration and redirection introduces considerable turbulence in the helium stratification.

\section{Introduction}

Incompressible turbulent round jets with density $\rho_{j}$ issuing into large, ideally infinite and quiescent domains with the same density are often referred to as building block flows and numerous experimenters have conducted different types of these measurements. An overview of the different phenomena and the physics in turbulent jets can be found, for example, in [1-3], and a recent review of experimental data is provided in $[4,5]$. However, quite often in real life applications, the jet flow is influenced by buoyancy $[4,6]$. For example, a jet of fluid with density $\rho_{j}$ which is directed into a volume of fluid of density $\rho_{a}$ with $\rho_{j} \neq \rho_{a}$ or a jet $\left(\rho_{j}\right)$ impinging onto a stable density stratification $\left(\rho_{a}\right)$ is often encountered during industrial processes. The sewage disposal through a pipe into a river or the sea is widely used in industry to mix the waste water with fresh water. Jets discharging into a two-layer stably stratified environment have received relatively little attention despite their fundamental nature and their potential practical significance. A ventilated room for example can naturally form a two-layer stratification and it is of interest to know how cold air injected from below mixes in this environment.

A jet with the density $\rho_{j}$ is called positively buoyant when the buoyancy force and the momentum have the same direction and the buoyancy force adds to the momentum such that the velocity decay-common for nonbuoyant jetsis partly compensated and for strong buoyancy forces, the jet might even accelerate. When the buoyancy and the momentum forces have opposite directions, the jet is called negatively buoyant; that is, the negative buoyancy decelerates the jet much faster compared with a nonbuoyant jet.

These phenomena also have relevance for nuclear safety analysis [7-12]. Three-dimensional computational fluid dynamics (CFD) codes, as well as advanced lumped parameter (LP) codes, are increasingly used for safety analysis to simulate transient containment conditions after various accident 
scenarios for present [13] and upcoming generations of nuclear power plants [14]. Consequently, the reliability of such codes must be tested against experimental data collected under prototypical thermal hydraulic conditions. Large scale test facilities [15] should be preferentially used to minimize distortional effects that might arise from geometrical scaling [11].

The experiment presented in the paper describes the interaction of vertical, upward projecting air-helium jet emerging from a tube below a helium-rich air layer. The experiment is carried out under isothermal and isobaric conditions and focuses onto the flow conditions at the tube exit, in addition to the mechanisms of entrainment of the helium stratification located just beneath the vessel dome. The experiment was conducted under conditions characterized by a jet which is initially buoyant and becomes negatively buoyant and decelerates when it reaches the helium stratification. Results depicting the mixing, transport, and transient helium stratification erosion are presented in terms of $2 \mathrm{D}$ PIV measurements which were used to measure the flow velocities at different locations and in particular at the interface between the helium layer and the upward impinging jet. Additional gas concentration measurements with a mass spectrometer are used to calculate density profiles which illustrate the erosion process of the density stratification.

It should be noted that the present experiment violates intentionally the Boussinesq approximations of the first and second kind. The Boussinesq approximation of the first kind is part of a series of simplifications in which the density variation in the continuity equation is neglected. The Boussinesq approximation in the momentum equation thus requires that the temperature or density differences in the fluid remain small. A $10 \%$ difference is normally regarded as the upper limit for this first approximation. The Boussinesq assumption of the second kind expresses the Reynolds stresses resulting from the RANS equations in terms of the mean strain rate:

$$
\overline{-u_{i}^{\prime} u_{j}^{\prime}}=v_{t}\left(\frac{\partial \overline{u_{i}}}{\partial x_{j}}+\frac{\partial \overline{u_{j}}}{\partial x_{i}}\right)
$$

with $v_{t}$ denoting the scalar, isotropic eddy viscosity [16]. It is noted that the introduction of high density gradients in horizontal stratified flows may lead to the violation of both kinds of Boussinesq assumptions simultaneously. The high density gradient violates the small density difference requirement, and the damping of the vertical velocity fluctuations in the vicinity of the stratification violates the eddy viscosity isotropy assumption. Consequently, besides the insight into fundamental physical mixing mechanisms in the presence of steep density gradients and an intended violation of the Boussinesq approximations, it is also expected that the conducted experiment poses some interesting computational challenges.

In Section 2 we give an introduction to the experimental facility, the instrumentation and the initial and boundary conditions. In Section 3 we discuss the experimental in-vessel results of the erosion process in the presence of a helium-rich stratification by means of velocity and temperature maps as well as concentration profiles. Some emphasis was put on the error calculation for the different measurement devices used.

\section{Experiment}

The vessel used for the experiments had an inner diameter of $4 \mathrm{~m}$ and a height of $8 \mathrm{~m}$; Figure 1 . The air-helium mixture forming the jet with density $\rho_{j}$ and the nominal tube exit velocity $v_{j, n}$ is injected through a tube which is positioned offaxis by $648 \mathrm{~mm}$ with respect to the axis of symmetry of the vessel. For the calculation of the nominal velocity we neglect the formation of boundary layers at the inner tube walls and assume a constant velocity across the horizontal tube exit plane. The injection tube has a $180^{\circ}$ bend $2200 \mathrm{~mm}$ below the tube exit. The straight tube past the bend has a length of $\approx 30 d_{t}$ with $d_{t}$ depicting the tube inner diameter which is long enough that possible disturbances introduced by the bend are removed by the turbulence due to the tube's high Reynolds number $\left(\operatorname{Re}_{j} \approx 20000\right.$; Table 1$)$. This will be confirmed by the tube exit velocity measurements described in a subsequent section. Consequently, the velocity profile at the tube exit shows top hat characteristics with boundary layers typical for turbulent pipe flows. In contrast to some past jet experiments where a smooth contraction nozzle at the tube exit was used to pronounce the top hat velocity profile by compressing the boundary layers, we used an injection tube with a constant nominal inner diameter of $d_{t}=75.3 \mathrm{~mm}$. Testing for the circularity of the tube, the actual inner diameter measured at the tube exit in two perpendicular planes amounted $d_{t, a}=$ 75.4 and $d_{t, b}=75.6 \mathrm{~mm}$ which is in good agreement with the nominal value used henceforth.

During the entire experiment the pressure was kept constant at about 0.994 bar absolute by venting of the air-helium mixture from the vessel via a funnel connected to a flexible hose-oriented downwards which was located at the bottom of the vessel; Figure 1. The injection tube exit is located $2995 \mathrm{~mm}$ above the bottom of the vessel. The coordinate system origin to describe the experiment is located at the bottom of the vessel and the light sheet for the PIV recordings coincides with the $x$ - $y$ plane; Figure 1 .

2.1. Instrumentation. For the $2 \mathrm{D}$ velocity measurements a particle image velocimetry (PIV) system was used. The PIV camera was mounted in front of the upper vessel window on a translation stage consisting of two goniometers and a rotation table. By vertically inclining the camera it was possible to record three different field-of-views (FOVs) to follow the erosion front progression of the helium layer. The three FOVs are depicted as PosA, PosB, and PosC in Figure 1.

An example of a raw PIV image recorded at position $B$ is shown in Figure 2(a). The image gives an impression of the jet-layer interaction zone. The seeded jet entering from below impinges onto the nonseeded helium-rich layer and penetrates the stratification. The corresponding instantaneous velocity field with some selected stream lines is pictured in Figure 2(b).

Olive oil, dispersed into small droplets by a spray nozzle, was used as seeding particles for the PIV technique. The oil 


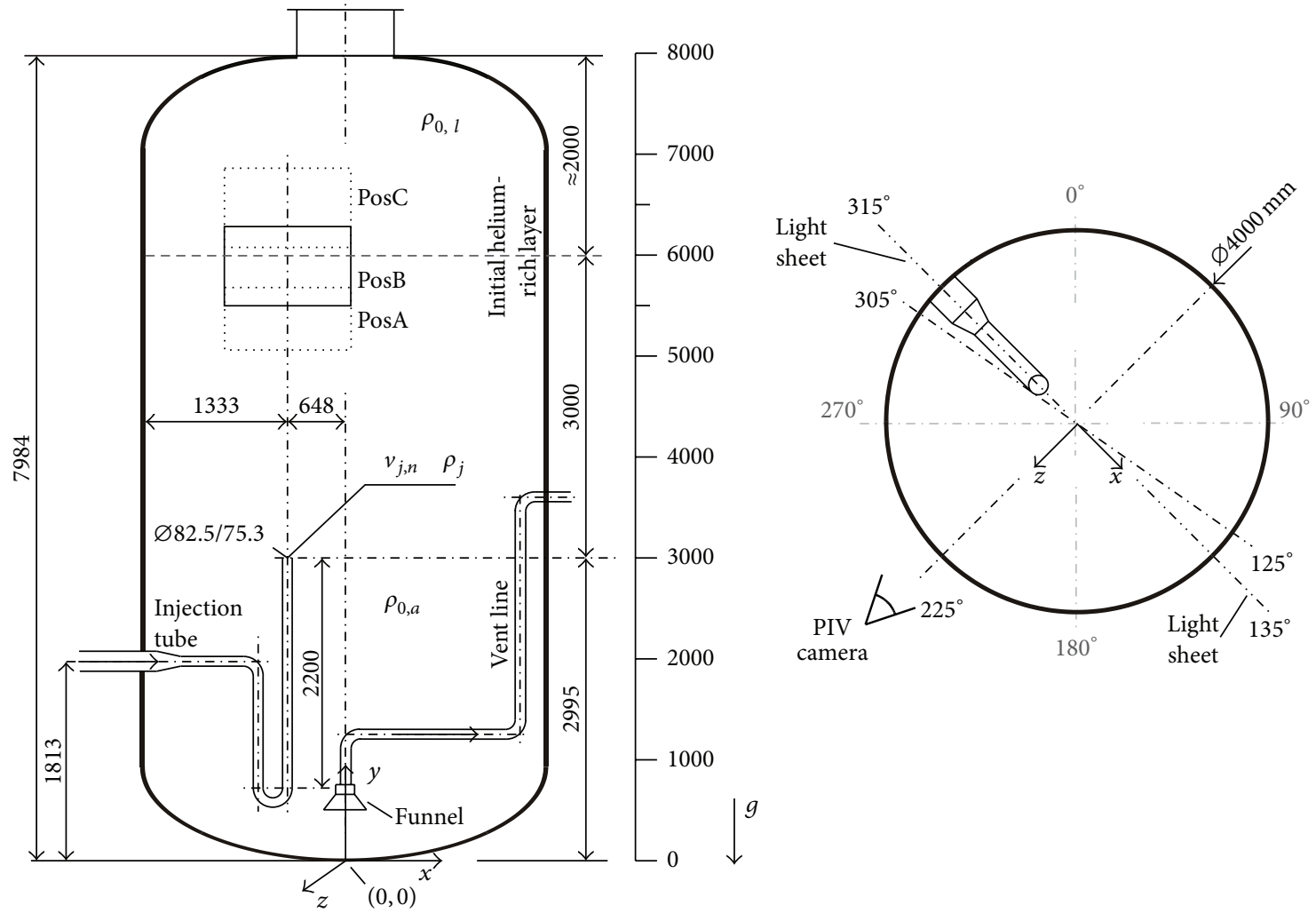

FIGURE 1: Schematic side and top view of the experimental facility with the initial helium-rich layer located in the vessel dome. The main dimensions are given in $\mathrm{mm}$.

TABLE 1: Main measured parameters for the PANDA N04 experiment conducted in the frame of the OECD/NEA PANDA benchmark. For the calculation of physical properties a nominal temperature of $T=22^{\circ} \mathrm{C}$ and the measured pressure of $p=0.994$ bar were used.

\begin{tabular}{|c|c|c|c|c|c|c|c|c|c|c|c|}
\hline \multirow[b]{2}{*}{ Number } & \multicolumn{7}{|c|}{ Jet } & \multicolumn{2}{|c|}{ amb. } & \multicolumn{2}{|c|}{ Layer } \\
\hline & $\begin{array}{r}\dot{m}_{\text {air }} \\
\mathrm{g} / \mathrm{s}\end{array}$ & $\begin{array}{r}\dot{m}_{\mathrm{he}} \\
\mathrm{g} / \mathrm{s}\end{array}$ & $\begin{array}{c}\dot{m}_{j}^{\dagger} \\
\mathrm{g} / \mathrm{s}\end{array}$ & $\begin{array}{c}\rho_{j} \\
\mathrm{~kg} / \mathrm{m}^{3}\end{array}$ & $\begin{array}{l}\bar{v}_{j, b} \\
\mathrm{~m} / \mathrm{s}\end{array}$ & $\mathrm{m}^{2} / \mathrm{s} \cdot 10^{-5}$ & $\operatorname{Re}_{j, b}$ & $\begin{array}{c}\rho_{o, a} \\
\mathrm{~kg} / \mathrm{m}^{3}\end{array}$ & $\begin{array}{c}\Delta \rho_{0, j a}{ }^{*} \\
\%\end{array}$ & $\begin{array}{c}\rho_{0, l} \\
\mathrm{~kg} / \mathrm{m}^{3}\end{array}$ & $\begin{array}{c}\Delta \rho_{0, j l} \mathbf{\wedge} \\
\%\end{array}$ \\
\hline N04 & 21.53 & 0.42 & 21.95 & 1.047 & 4.67 & 1.81 & 20000 & 1.173 & $\approx+11$ & 0.772 & $\approx-36$ \\
\hline
\end{tabular}

${ }^{\dagger}$ Calculated with $\dot{m}_{j}=\dot{m}_{\text {air }}+\dot{m}_{\text {he }}$

${ }^{\ddagger}$ Calculated as $\Delta \rho_{0, j a}=\left(\rho_{o, a}-\rho_{j}\right) / \rho_{o, a} \cdot 100$

${ }^{\Delta}$ Calculated as $\Delta \rho_{0, j l}=\left(\rho_{o, l}-\rho_{j}\right) / \rho_{0, l} \cdot 100$

particles were injected into the air stream that was directed into the vessel through the injection line.

The PIV system setup for the in vessel measurements provides $2 \mathrm{D}$ velocity fields with an acquisition rate of $5 \mathrm{~Hz}$ (the PIV setup for the tube exit measurements will be described in a subsequent section). For the calculation of statistical quantities 1024 image pairs were averaged which results in an overall averaging time of $204.8 \mathrm{~s}$. The PIV system consisted of a Quantel Twins B double pulse laser with a maximum output energy of $380 \mathrm{~mJ}$ and a double frame CCD camera type Imager Pro X which is identical to the PCO.1600 camera, with a resolution of $1600 \times 1200$ pixel. After calibration of the images a resolution of $0.715 \times 0.715 \mathrm{~mm}^{2} /$ pixel was achieved which corresponds to an effective spatial resolution of $11.5 \times 11.5 \mathrm{~mm}^{2}$ for the velocity field. The absolute statistical error with confidence band of $\pm 95 \%$ for the mean velocity calculation is $\epsilon= \pm 0.014 \mathrm{~m} / \mathrm{s}$ on average.

The gas concentration was measured in the facility by means of two mass spectrometers (MassSpec). Gas was continuously sampled and sent to the MS systems through capillaries having a tip inner diameter of $0.2 \mathrm{~mm}$ which renders possible leak rates during the MassSpec measurements negligible. Each of these tubes is equipped with a thermocouple to record the temperature of the gas at the capillary inlet associated. The MS measurement is sequential and only one line can be selected at a time via a multiport rotating valve. When selected, the sampled gas is sent into a quadrupole mass spectrometer which gives the partial pressure of the selected gas stream (air and helium). From these partial pressures and the temperatures the molar fractions 


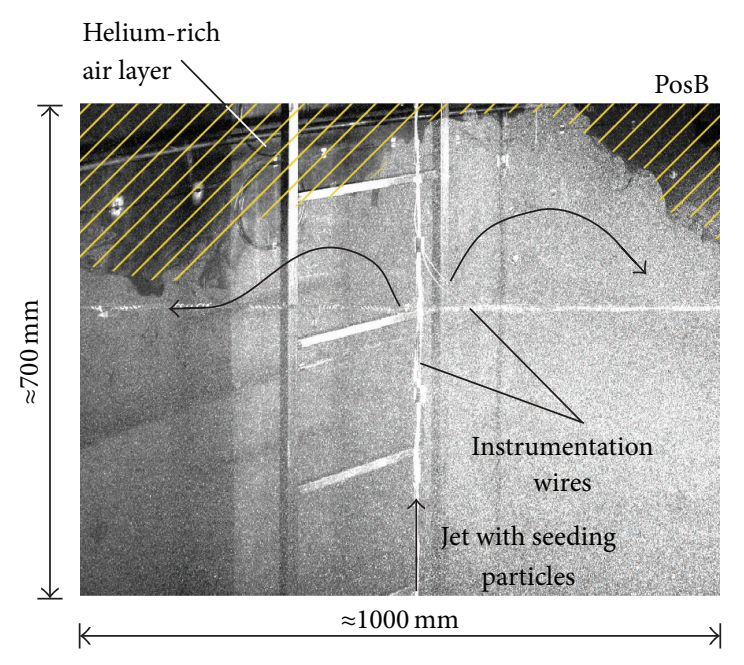

(a)

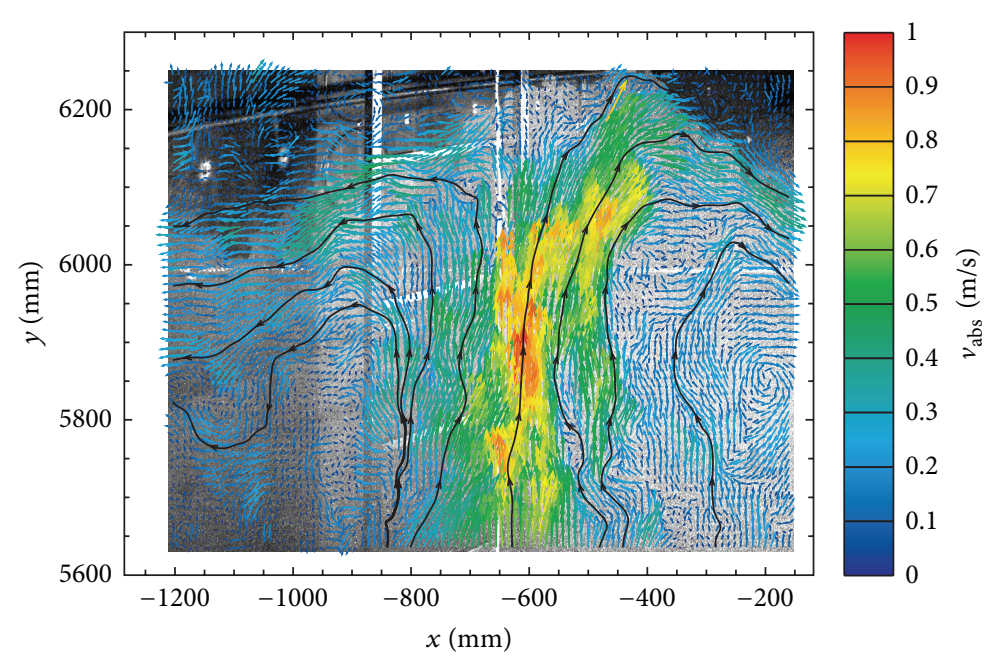

(b)

FIgURE 2: Example of a PIV raw image recorded for position B showing the seeded jet in the lower part, the nonseeded helium-rich air layer at the top, and parts of the instrumentation wires (a) and the corresponding instantaneous velocity field with selected stream lines (b). Dimensions are given in $\mathrm{mm}$.

and finally the densities were calculated. The MS capillaries as well as the thermocouples are mounted on instrumentation wires throughout the entire vessel. One horizontal and one vertical instrumentation wire are visible in the PIV recording pictured in Figure 2(a). The measurement error of the MS system is $1 \%$ absolute.

For the measurement of gas temperatures for the present experiments Type- $\mathrm{K}$ thermocouples (TC) with a diameter of $1 \mathrm{~mm}$ were used. Three of these thermocouples out of the total batch of 266 used in the present experiments were calibrated at the "Deutscher Kalibrierdienst" at the "Physikalisch-Technische Bundesanstalt (PTB)" in Braunschweig (Germany) for the temperature range 40 to $200^{\circ} \mathrm{C}$. Based on the deviation of the actual reading from the set value, a common calibration curve to compensate for this offset was derived from all three TCs and applied to the entire set (266 TCs). With the compensation of the offset and from the calibration at the PTB we calculated an error of $\epsilon_{t c}= \pm 0.7^{\circ} \mathrm{C}$ with a confidence band of $\pm 95 \%$ for the temperature reading.

2.2. Initial and Boundary Conditions. Prior to the test, stratified air/helium conditions have been created in the test vessel. A helium-rich air layer with density $\rho_{0, l}$ occupies the region $y>6000 \mathrm{~mm}$ (Figure 1), while pure air with density $\rho_{0, a}$ fills the region below $y=5000 \mathrm{~mm}$. Between the air filled lower part of the vessel and the helium-rich air layer, one finds a transitional region, $5000<y<6000 \mathrm{~mm}$, where the helium content increases continuously.

The measured helium and air molar fractions at time $t=0$ as a function of elevation are displayed in Figure 3. This figure has been compiled from MassSpec measurements taken principally along the axis of the vessel. However, a number of off-axis measurements have also been included to

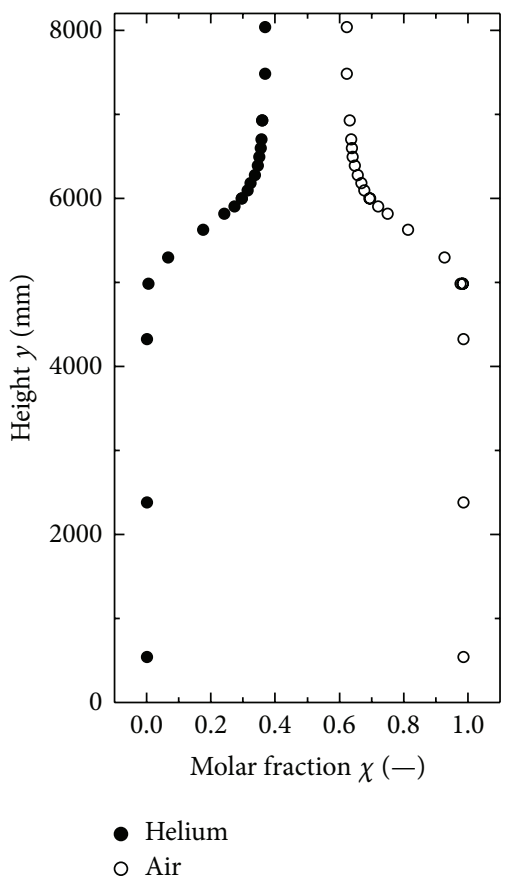

FIGURE 3: Initial air and helium molar fraction as a function of height in the vessel.

demonstrate the flatness of the initial horizontal stratification. A table of the MassSpec measurement points can be found in [22] in Table A5. Note the near-zero concentration level of helium for $y<5000 \mathrm{~mm}$ (Figure 3) and the nonlinear increase with height to around 0.37 helium molar fraction at elevation $y=8030 \mathrm{~mm}$. The measured and the nominal 
parameters of the entire experimental series can be found in Table 1 . The deviation from the nominal values was below $1 \%$. For example, the nominal mass flow rate was $22 \mathrm{~g} / \mathrm{s}$, while the measured mean over the entire experiment was calculated as $21.95 \mathrm{~g} / \mathrm{s}$. The air mass flow rate was measured using a thermal mass flow meter having an accuracy of $1.5 \%$ of the measured value according to the manual. The mass flow rate was averaged of $6588 \mathrm{~s}$ (3294 data points sampled with $0.5 \mathrm{~Hz}$ ) with results in a mean flow rate of $21.53 \mathrm{~g} / \mathrm{s}$ and a standard deviation of $0.23 \mathrm{~g} / \mathrm{s}$, respectively.

The mass flow rate of helium was also measured with a thermal mass flow meter and the sampling frequency and the sampling time was the same. The helium mass flow meter was calibrated at the manufacturer's facility with air. Since the conversion from the air calibration into the helium mass flow rate involved some uncertainties, we have decided to use different conversion methods to assess the important helium mass flow rate in the jet. (1) We used the manufacturer calibration along with the manufacturer supplied software for the air to helium conversion. (2) We used the manufacturer's calibration together with the ratio of the specific heats for air and helium, respectively. (3) We compared the flow meter measurement against the calculated flow rate necessary to increase the pressure in a large vessel by a certain amount. (4) We used the available MassSpec data to calculate the helium mass flow in an air-helium mixture, while the air flow rate was measured with the standard thermal mass flow meter. Finally, we sent out the mass flow meter for a separate nonmanufacturer calibration lab and used (5) the new calibration coefficients and (6) the new calibration coefficients together with the specific heat ratios. Combining all six methods and using statistical calculations, the mean helium mass flow rate in the jet amounts to $0.42 \mathrm{~g} / \mathrm{s}$ and the error was $\pm 0.022 \mathrm{~g} / \mathrm{s}$ with a confidence limit of $99 \%$.

For further calculations the following physical properties calculated according to Lemmon et al. [23] were used: air at $p=0.994$ bar and $T=22^{\circ} \mathrm{C}$ has a density of $\rho_{\text {air }}=1.1735 \mathrm{~kg} /$ $\mathrm{m}^{3}$ and a kinematic viscosity of $v_{\text {air }}=1.5598 \cdot 10^{-5} \mathrm{~m}^{2} / \mathrm{s}^{2}$ and for helium we obtain $\rho_{\text {he }}=0.16205 \mathrm{~kg} / \mathrm{m}^{3}$ and $\nu_{\text {he }}=12.162$. $10^{-5} \mathrm{~m}^{2} / \mathrm{s}^{3}$.

The jet Reynolds number $\operatorname{Re}_{j, b}$ at the tube exit

$$
\operatorname{Re}_{j, b}=\frac{v_{j, b} \cdot d_{t}}{v_{j}}
$$

was calculated using the tube's nominal inner diameter $d_{t}$ and the bulk velocity $v_{j, b}$. Since the flow rate is kept constant during the entire experiment, this Reynolds number characterizes the momentum flux of the jet injected. To characterize the initial buoyancy we use the source densimetric Froude number:

$$
\operatorname{Fr}_{0}=\frac{v_{j, b}}{\sqrt{\left(\rho_{o, a}-\rho_{j}\right) / \rho_{o, a} \cdot g \cdot d_{t}}}
$$

with the gravity acceleration $g$ and we obtain $\mathrm{Fr}_{0}=15.6$ which indicates a "jet-like" flow in the near field of the injection tube. To characterize the initial stratification strength and the buoyant conditions we define two (initial) density differences. The density difference between the jet and the ambient:

$$
\Delta \rho_{0, j a}=\frac{\rho_{o, a}-\rho_{j}}{\rho_{o, a} \cdot 100}
$$

and the density difference between the helium-rich layer and the jet:

$$
\Delta \rho_{0, j l}=\frac{\rho_{o, l}-\rho_{j}}{\rho_{0, l} \cdot 100} .
$$

Initially, right after the tube exit and for $t=0 \mathrm{~s}$, the vertical jet experiences a positively buoyant force; the velocity decay is partly compensated or the jet even accelerates as indicated by $\Delta \rho_{0, j a} \approx 11 \%$. After a certain distance, when the jet approaches the helium-rich layer, the "ambient" density continuously decreases such that the initially buoyant jet becomes increasingly negatively buoyant-as indicated by $\Delta \rho_{0, j l} \approx-36 \%$ - when penetrating the helium-rich layer and the axial velocity decays very rapidly; the latter calculation neglects the entrainment of ambient gas into the jet on its way from the tube orifice to the helium-rich layer and the corresponding density increases. Both density differences depict the nonvalidity of the Boussinesq approximation of the first kind at the beginning of the experiment as discussed in the introduction. The time dependent buoyancy in the course of the experiment will be discussed in a subsequent section.

2.3. Tube Exit Velocities. PIV measurements of the instantaneous values of the Cartesian velocity components $(u, v)$ of the gas mixture emerging from the injection pipe outlet were made. The PIV FOVs were taken over the pipe's internal cross-section in five horizontal planes, beginning $6 \mathrm{~mm}$ above the injection pipe outlet. From the horizontal measurement planes, velocity profiles were extracted at $y=7.3 \mathrm{~mm}$ above the outlet; a position corresponding the closest approach to $y / d_{t}=0.1$. A schematic of the arrangement is shown in Figure 4. To gain better access, these measurements were taken with the injection line removed from the PANDA vessel, though still connected to the same helium and air supply lines used in the actual test. The laser sheet was positioned at the five (5) lines $\mathrm{A}-\mathrm{E}$, at $15 \mathrm{~mm}$ spacing, over the cross-section of the injection pipe outlet, as indicated in Figure 4 . The gas mixture in this case was not preheated; the ambient temperature was estimated at $15^{\circ} \mathrm{C}$.

The PIV system setup for the tube exit measurements provides $2 \mathrm{D}$ velocity fields with an acquisition rate of $7.5 \mathrm{~Hz}$. For the calculation of statistical quantities 4096 image pairs were averaged. The PIV system consisted of a Litron nano L double pulse laser with a maximum output energy of $200 \mathrm{~mJ}$ and the same camera used for the in-vessel measurements. The light sheet thickness was approximately $1 \mathrm{~mm}$.

The optical recording system consisting of the camera and a Nikon lens (AF Nikkor, $f=50 \mathrm{~mm}$; the aperture was set to $f_{\#}=4$ ) was operated in the diffraction limit; that is, the particle image size becomes independent of the physical particle size resulting in particle image sizes larger 


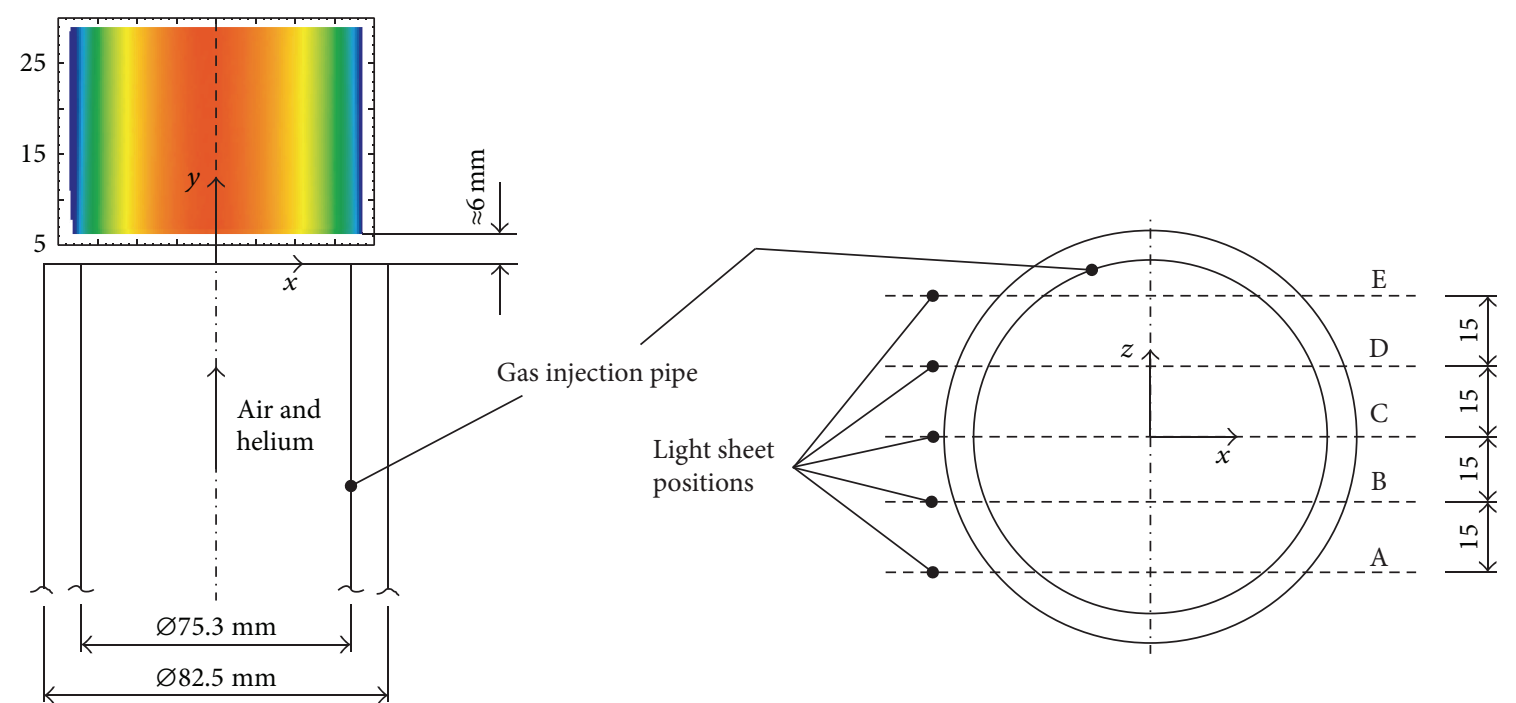

FIGURE 4: Relative positions of the FOVs of the PIV velocity measurements at the exit of the injection line; note the local coordinate system used for the measurement planes A-E which differs from the one given in Figure 1.

than 3 pixels on average. According to the recommendations in Raffel et al. [24] this is sufficient to minimize so-called peak-locking. The base analysis was performed with $\mathrm{DaVis}$ 8.1 and the extended analysis with in house written MATLAB routines. After calibration of the images, a resolution of $0.088 \times 0.088 \mathrm{~mm}^{2} / \mathrm{pixel}^{2}$ was obtained which corresponds to an effective spatial resolution of $0.53 \times 0.53 \mathrm{~mm}^{2}$ for the velocity field. A water/polyethylene-glycol mixture (10 : 1), dispersed into small droplets by an atomizer, was used for the seeding particles for the PIV measurements. The particles were injected into the air stream approximately $5000 \mathrm{~mm}$ $\left(\approx 60 d_{t}\right)$ upstream of the injection pipe exit. The primary particles have an approximate diameter of $D_{10} \approx 4 \mu \mathrm{m}$. While the helium for the air-helium mixture was dry, the air was drawn from that available at the pump inlet and was not dried before injection into the pipe. Consequently the air reflects the humidity of the atmosphere in the PANDA building at the time of the test. This results in an approximate relative humidity of $60 \%$ for the air-helium mixture. It is expected that a part of the water from the water/polyethylene-glycol droplets evaporates already in the pipe and that the droplets have a final size of $D_{10} \approx 1.5 \mu \mathrm{m}$. Using a simplified version of the Basset-Boussinesq-Oseen equation (BBO) for the density ratio $\rho_{p} / \rho_{f} \gg 1$ between particle $(p)$ and fluid $(f)$, see Raffel et al. [24],

$$
\frac{u_{p}}{u_{f}}=\frac{1}{\sqrt{1+\omega_{f}^{2} \tau_{0}^{2}}} \text { with } \omega_{f}=2 \pi f_{f}, \quad \tau_{0}=\frac{\rho_{p} d_{p}^{2}}{18 \eta_{f}}
$$

with the amplitude ratio $u_{p} / u_{f}$ between the particle response to the amplitude of the fluid motion, the expected frequency of the fluid motion $f_{f}$, and the particle time constant $\tau_{0}$, this allows for the calculation of the amplitude response of such particles; Figure 5. Equation (6) resembles a second order, low pass filter response for the ratio of the velocity amplitudes.

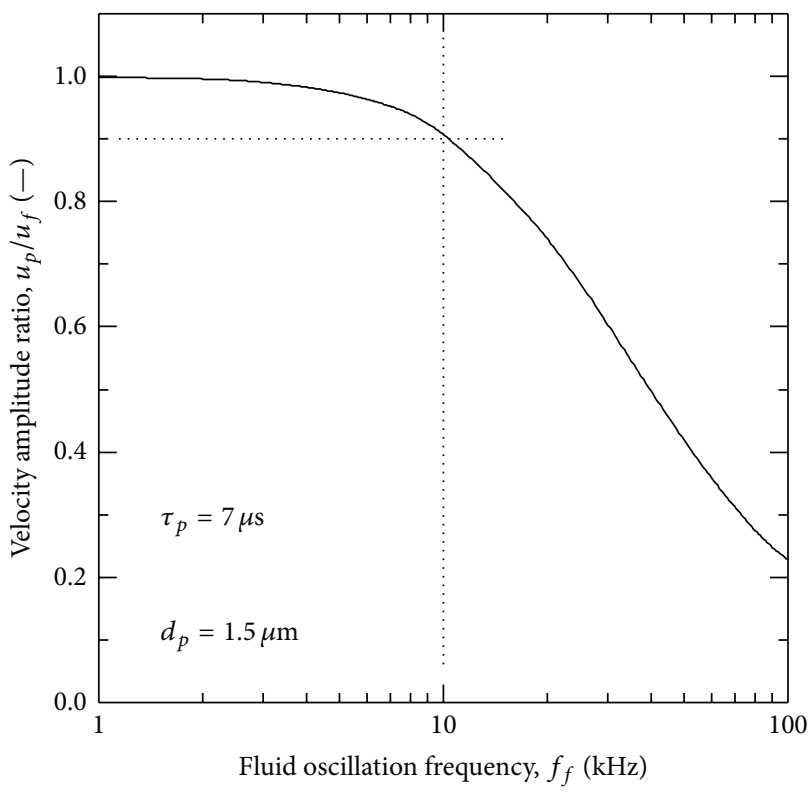

FIGURE 5: Velocity amplitude ratio between particle and fluid motion as a function of fluid motion frequency.

Using an order of magnitude argument, it follows that the particles with time constant $\tau_{p}=7 \mu$ s under consideration can resolve fluid motions up to a frequency of $10 \mathrm{kHz}$ if the minimum allowable amplitude ratio amounts to $u_{p} / u_{f} \approx 0.9$. On the other hand, the maximum expected frequencies of the smallest flow structures close to or in the inertial subrange can be estimated according to Albrecht et al. [25]:

$$
f_{\max , f}=\frac{\bar{u}_{f}}{2 \pi L_{u}} \operatorname{Re}^{3 / 4}
$$


with a characteristic macrolength scale $L_{u}$ for the velocity field. Using the inner pipe diameter for this length scale $L_{u}=d_{t}=75.3 \mathrm{~mm}, \bar{u}_{f}=\bar{v}_{j, n}=4.67 \mathrm{~m} / \mathrm{s}$ and $\operatorname{Re}=$ $\mathrm{Re}_{j}=20000$, Table 1 , results in $f_{\text {max }, f} \approx 16 \mathrm{kHz}$ which is in good agreement with the frequency $(10 \mathrm{kHz})$ the particles can resolve. Additionally, an integral time scale for the pipe flow can be estimated from $T_{u}=L_{u} / \bar{v}_{j, n}=0.016 \mathrm{~s}$ which allows estimating the necessary time separation required such that successive measurements decorrelate; that is, they become statistically independent: $2 T_{u}=0.032 \mathrm{~s}$. This time corresponds to a maximum allowable sampling frequency of $\approx 31 \mathrm{~Hz}$ which is above the chosen frequency of $7.5 \mathrm{~Hz}$. It can be concluded that the instantaneous velocity fields are statistically independent and each velocity field contributes to the convergence of the statistical quantities calculated.

The velocity profiles and the calculated statistics shown in Figures 6 and 7 were extracted for $y / d_{t}=0.097$ above the tube exit and show the results for the vertical $v$-component (upwards in the $y$ direction) as well as the lateral $u$-component (horizontally in the $x$ direction) for the five measurement planes (Figure 4). As a consequence of technical restrictions, and time constraints, it was not possible to measure the third velocity component ( $w$-component, horizontally in the $z$ direction). However, the assumption of axis-symmetric outlet conditions is expected to prevail in this test. The radial distances ( $x$ direction) were normalized with the nominal pipe diameter $d_{t}=75.3 \mathrm{~mm}$ and the velocity results with the center line $(x=0)$ axial mean velocity $v_{c}=5.35 \mathrm{~m} / \mathrm{s}$.

From the measured instantaneous velocity components $u_{i}$ (and $v_{i}$ ), mean $\bar{u}(\bar{v})$, and time averaged standard deviations $u^{\prime}\left(v^{\prime}\right)$ of the pipe exit velocity data have been calculated. The procedure used was as follows:

$$
\begin{gathered}
\bar{u}=\frac{1}{N} \sum_{i=1}^{N} u_{i}, \\
u^{\prime}=\sqrt{\frac{1}{N} \sum_{i=1}^{N}\left\{u_{i}-\bar{u}\right\}^{2} .}
\end{gathered}
$$

Additionally we calculate the Reynolds stresses $\overline{u^{\prime} u^{\prime}}\left(\overline{v^{\prime} v^{\prime}}\right.$ and $\left.\overline{u^{\prime} v^{\prime}}\right)$ and the turbulent kinetic energy $k$ as follows:

$$
\begin{gathered}
\overline{u^{\prime} u^{\prime}}=\frac{1}{N} \sum_{i=1}^{N}\left\{u_{i}-\bar{u}\right\}^{2}, \\
\overline{u^{\prime} v^{\prime}}=\frac{1}{N} \sum_{i=1}^{N}\left\{u_{i}-\bar{u}\right\}\left\{v_{i}-\bar{v}\right\}, \\
k=\frac{1}{2}\left\{2 \cdot \overline{u^{\prime} u^{\prime}}+\overline{v^{\prime} v^{\prime}}\right\} .
\end{gathered}
$$

Here $N$ is the number of instantaneous velocity recordings used in forming the average: these were taken from 4096 statistically independent samples, obtained at a sampling frequency of $7.5 \mathrm{~Hz}$, which corresponds to an overall averaging time of $546 \mathrm{~s}$ (9.1 $\mathrm{min})$. Analogous definitions apply for another velocity component $v$. To calculate $k$ with (11) we made use of $\overline{u^{\prime} u^{\prime}}=\overline{w^{\prime} w^{\prime}}$ (assumption of axis-symmetry), since the third velocity component $w$ was not accessible. For the normalized axial mean velocity profiles $\bar{v}\left(x / d_{t}\right) / v_{c}$ (Figure 6(a)) measured in five planes, we find a good agreement between planes $\mathrm{A}-\mathrm{E}$ and $\mathrm{B}-\mathrm{D}$ displaced by 30 and $15 \mathrm{~mm}$, respectively, with respect to plane $\mathrm{C}$ indicating a rotational symmetric velocity profile across the entire tube exit. This holds also true for the other statistics; Figures 6 and 7. It should be noted that in the core region of the jet $(-0.1<$ $\left.x / d_{t}<0.1\right)$ a nonzero value for the mean lateral velocity component $\bar{u}$ has been measured for all the measurement planes (Figure 6(c)). This is considered as a consequence of the slightly rotated camera angle with respect to the jet axis, resulting in a "virtual redistribution" of the vertical velocity in the lateral velocity direction, which should be zero in the core of the jet. If the central measurement plane $\mathrm{C}$ is regarded as representative $(u=0.013 \mathrm{~m} / \mathrm{s} ; v=5.35 \mathrm{~m} / \mathrm{s})$, this would indicate a camera inclination of $0.14^{\circ}$. Removing this bias, the "true" axial velocity would then be $5.35001 \mathrm{~m} / \mathrm{s}$. To test for the similarity of the $\bar{v}$ profile at the tube exit with turbulent pipe flow, we applied two fit functions to the PIV data (Figure 8); a sine fit approach according to Chant [26]

$$
\frac{v}{v_{c}}=\left[\sin \left\{\frac{\pi}{2}\left(1-\left|2 \frac{x}{d_{t}}\right|^{1 / 2}\right)\right\}\right]^{1 / 2}
$$

as well as the classical $1 / 7$ power law

$$
\frac{v}{v_{c}}=\left|2 \frac{x}{d_{t}}\right|^{1 / 7} \text {. }
$$

Although our experimental data agree reasonably well with the $1 / 7$ power law, the sine fit (12) gives a much better approximation of the data. No attempt was made to improve the performance of (13) by adjusting the exponent $1 / n$.

For the statistical error estimate, reference should be made to Figure 6. Neglecting the boundary layers, an average value for the mean axial velocity would typically be of magnitude $\bar{v}=4.8 \mathrm{~m} / \mathrm{s}$ in plane $\mathrm{C}$, with a standard deviation across the profile of around $v^{\prime} \approx 0.4 \mathrm{~m} / \mathrm{s}$. Applying statistical calculus for statistically independent samples [27] the twosided uncertainty, with $95 \%$ confidence level, is estimated at $\varepsilon(v)= \pm 0.0122 \mathrm{~m} / \mathrm{s}$ for the mean vertical velocity $\bar{v}$ and nondimensionalised $\varepsilon(\bar{v}) / v_{c}= \pm 0.0022$. Analogous estimates apply also for the lateral velocities and result in $\varepsilon(\bar{u})=$ $\pm 0.0092 \mathrm{~m} / \mathrm{s}\left(\varepsilon(\bar{u}) / v_{c}= \pm 0.0017\right)$. Since the error for the standard deviations $\left(v^{\prime} \approx 0.4 \mathrm{~m} / \mathrm{s}\right.$ and $\left.u^{\prime} \approx 0.3 \mathrm{~m} / \mathrm{s}\right)$ is nonsymmetric [27], the two-sided uncertainty levels can be estimated as follows:

$$
\begin{aligned}
v^{\prime}=0.4_{-0.0084}^{+0.0088} \mathrm{~m} / \mathrm{s}, & u^{\prime}=0.3_{-0.0063}^{+0.0066} \mathrm{~m} / \mathrm{s}, \\
\frac{v^{\prime}}{v_{c}}=0.074_{-0.0015}^{+0.0016}, & \frac{u^{\prime}}{v_{c}}=0.056_{-0.0011}^{+0.0012} .
\end{aligned}
$$

A review of previous experiments investigating the scalar field either close to the tube exit or in fully turbulent pipe flow has been undertaken to assess the present boundary conditions. Despite the importance of the inlet conditions onto 


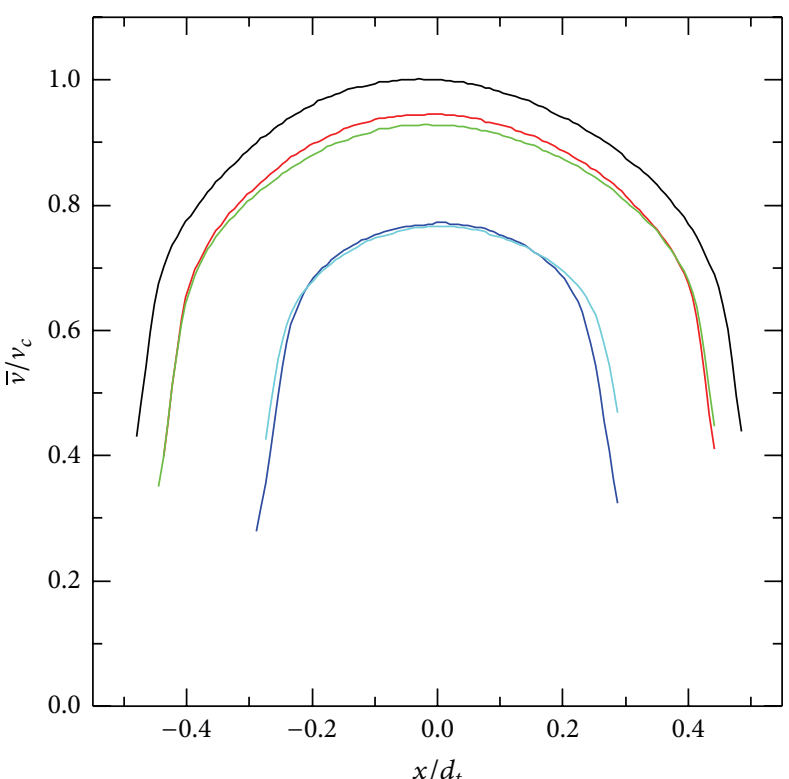

(a)

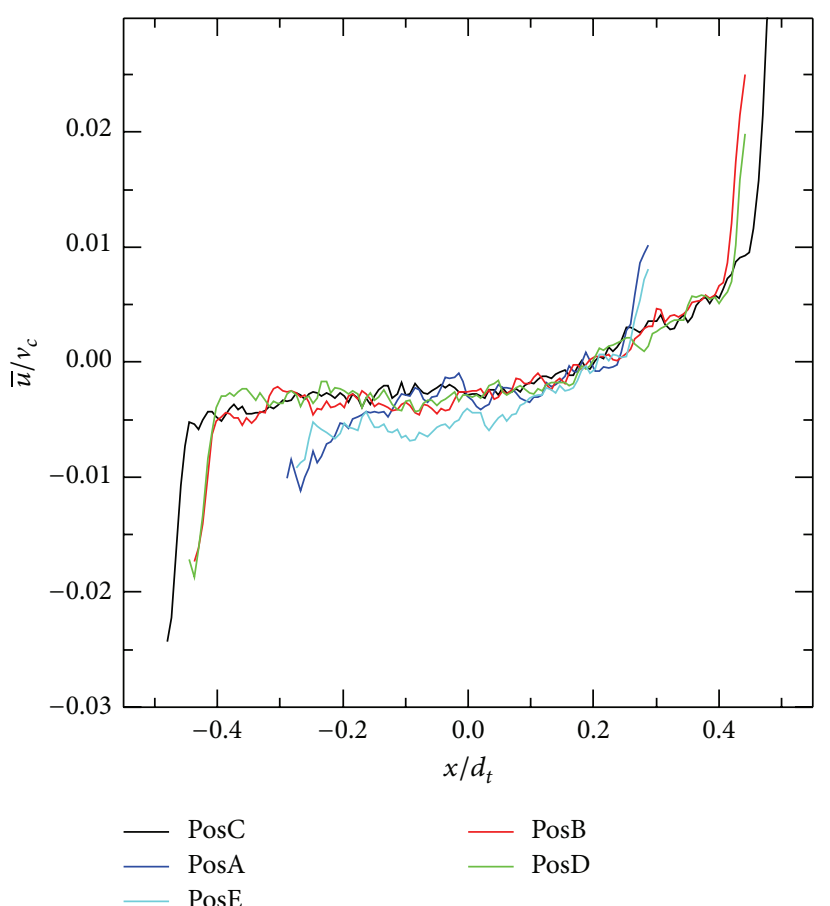

(c)

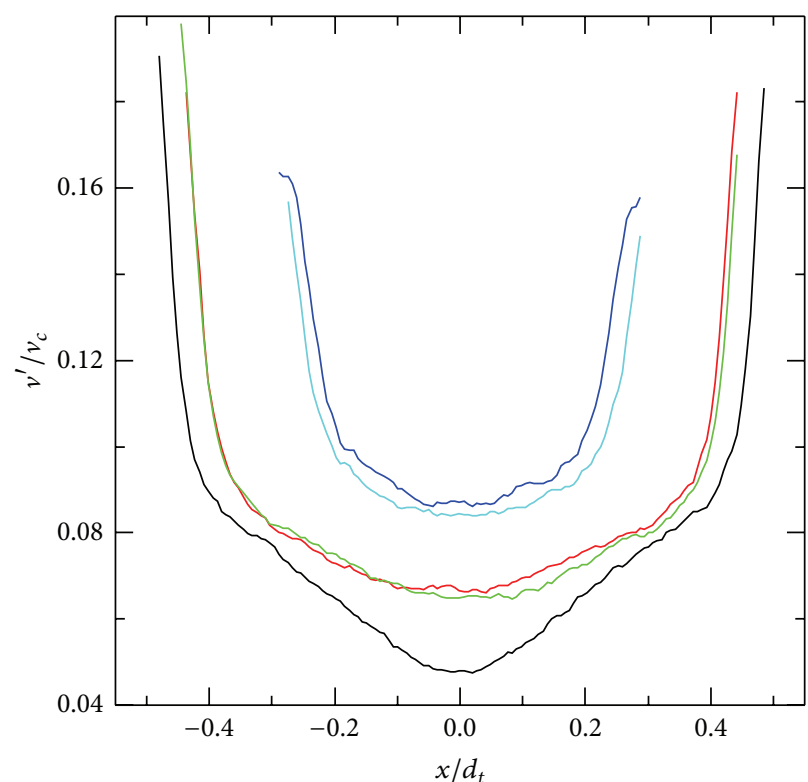

(b)

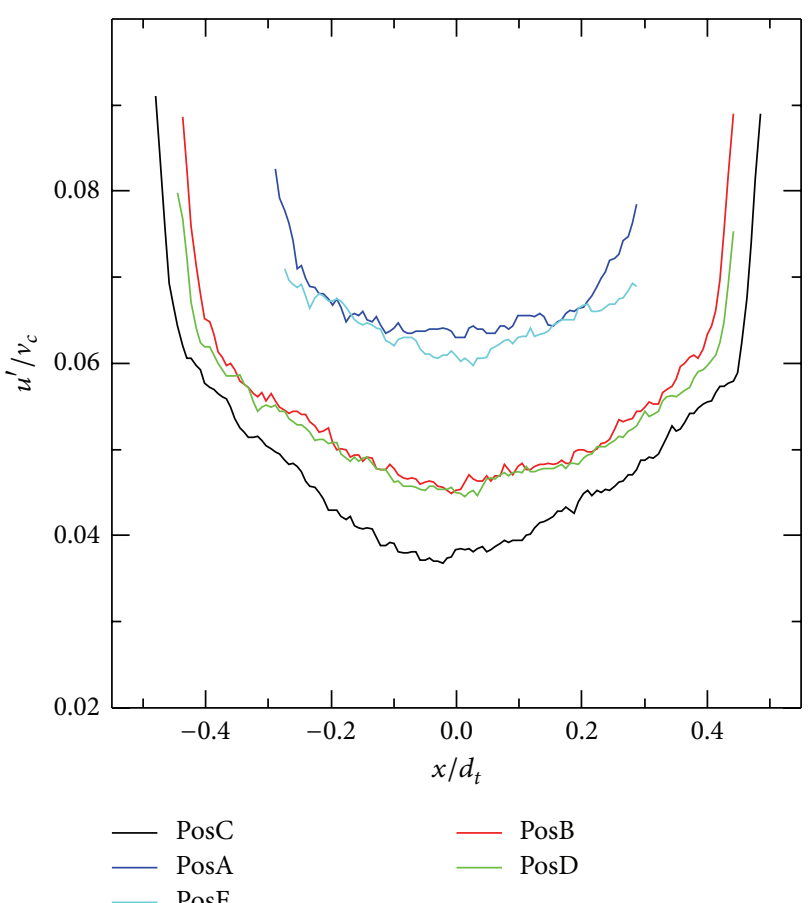

(d)

Figure 6: Normalized mean axial $\bar{v}$ and lateral $\bar{u}$ velocity profiles ((a) and (c)) and the corresponding $v^{\prime}$ and $u^{\prime}((\mathrm{b})$ and (d)) for the measurement planes A-E (Figure 4 ) at $y / d_{t}=0.097$ above the injection pipe exit.

the developing flow field in the near field $\left(0<y / d_{t} \leqslant 8\right)$ as well as for intermediate distances $\left(8 \lesssim y / d_{t} \leqslant 20\right)$, [17, 28], measurements close to the tube exit are surprisingly scarce. Turbulent pipe flow results have been included since the jet originates from a pipe and it is expected that the flow field at least in the core of the jet still shows pipe flow characteristics for the short distance past the pipe exit considered here. Except for the data selected from Eggels et al. [21] and
Boguslawski and Popiel [19] size, the experiments have been chosen with bulk Reynolds numbers as close as possible to the present experiment. The results from Boguslawski and Popiel [19] with the higher Reynolds number of 51000 were chosen since they provide results for $u^{\prime}$ at the tube exit. The data from the literature were digitized and renormalized according to the method introduced for the present paper, since different authors usually use different normalization 


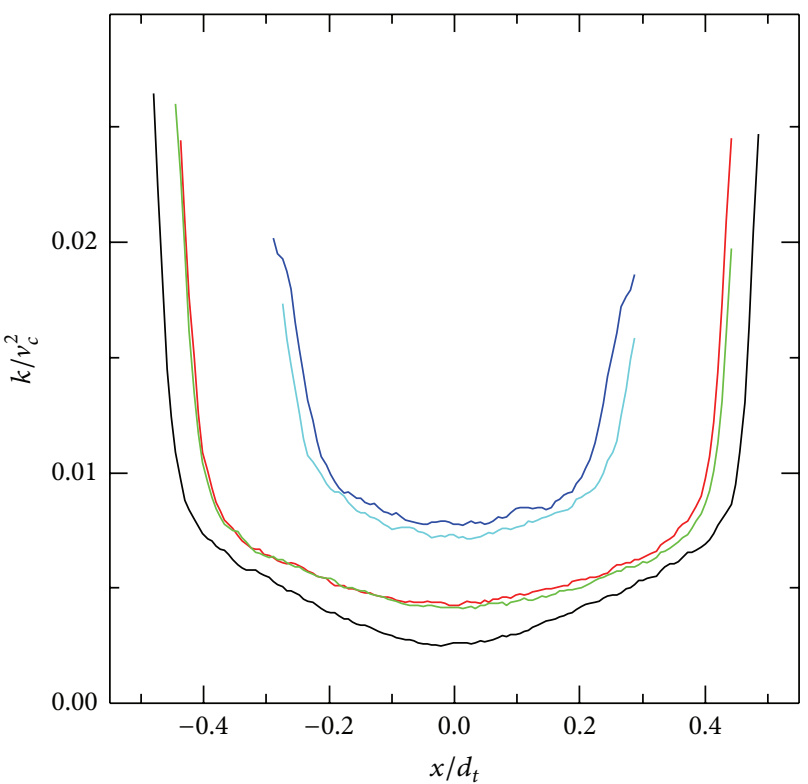

(a)

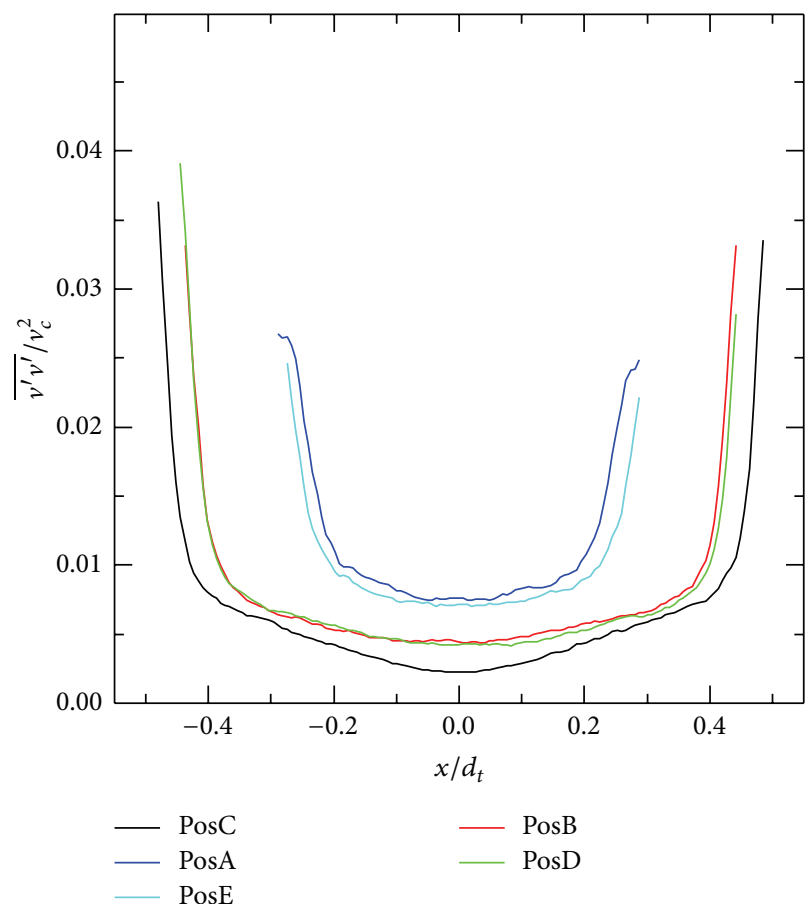

(c)

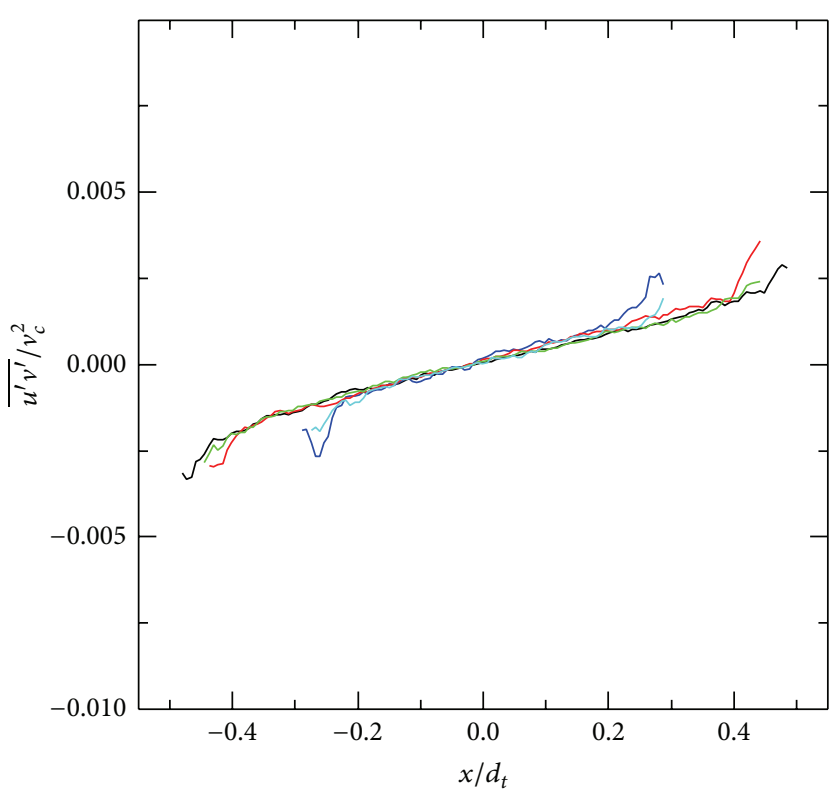

(b)

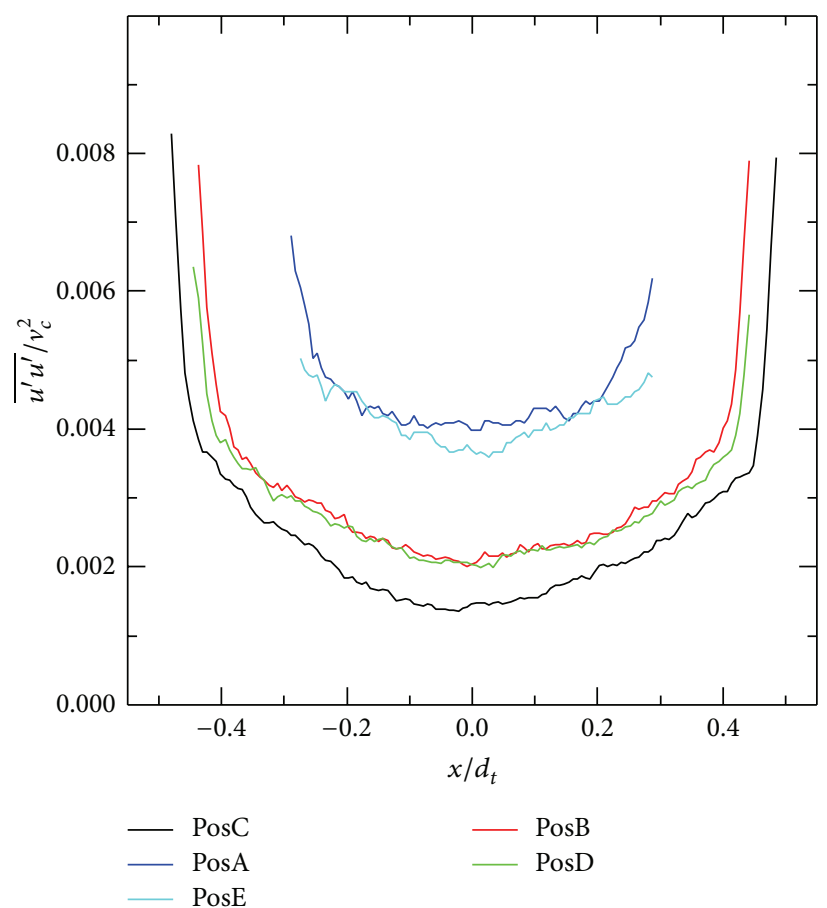

(d)

Figure 7: Normalized turbulent kinetic energy $k$ calculated with (11) (a) and Reynolds stresses $\overline{u^{\prime} v^{\prime}}, \overline{v^{\prime} v^{\prime}}$, and $\overline{u^{\prime} u^{\prime}}$ ((b), (c), and (d)) for the measurement planes A-E (Figure 4) at $y / d_{t}=0.097$ above the injection pipe exit.

schemes. An overview of the main characteristics for the selected experiments can be found in Table 2 and the results of the comparison for $\bar{v}, v^{\prime}, u^{\prime}$ are presented in Figures 9 (a) to $9(\mathrm{c})$. For the normalized mean axial velocity $\bar{v} / v_{c}$ as a function of radial distance $x / d_{t}$ (Figure 9(a)), we find a good agreement between our results and those quoted in the literature, irrespective of the experimental setup-pipe exit or turbulent pipe flow-and despite of a weak scatter the data which becomes larger when approaching either the mixing zone (pipe exit) or the pipe wall (pipe flow), $0.4<x / d_{t}<0.5$.

This finding supports to a certain extent the approach of also comparing velocity fluctuations measured in bounded (turbulent pipe flow) with unbounded (tube exit) experiments. When examining the normalized axial velocity fluctuations $v^{\prime} / v_{c}$, see Figure 9(b), our measurements agree better with pipe flow results rather than other jet experiments; 
TABLE 2: Flow properties for the jet exit and pipe measurements from selected references used to compare with the present experiments.

\begin{tabular}{|c|c|c|c|c|c|c|c|c|}
\hline Reference & Fluid & Location & Meas. techn. & $\operatorname{Re}_{j, b}$ & $y / d_{t}$ & $\bar{v}$ & $v^{\prime}$ & $\overline{u^{\prime}}$ \\
\hline Present & Air and $\mathrm{He}$ & Tube exit & PIV & 20000 & 0.097 & $\sqrt{ }$ & $\sqrt{ }$ & $\sqrt{ }$ \\
\hline Mi et al. [17] & Air & Tube exit & Cold wire & 16000 & 0.05 & $\sqrt{ }$ & $\sqrt{ }$ & - \\
\hline Papadopoulos and Pitts [18] & Air & Tube exit & Hot wire & 17500 & 0.16 & $\sqrt{ }$ & $\sqrt{ }$ & - \\
\hline Boguslawski and Popiel [19] & Air & Tube exit & Hot wire & 51000 & $\approx 0$ & $\sqrt{ }$ & $\sqrt{ }$ & $\sqrt{ }$ \\
\hline den Toonder and Nieuwstadt [20] & Water & In pipe & LDA & 17800 & - & $\sqrt{ }$ & $\sqrt{ }$ & $\sqrt{ }$ \\
\hline Eggels et al. [21] & Water & In pipe & PIV & 5450 & - & $\sqrt{ }$ & $\sqrt{ }$ & $\sqrt{ }$ \\
\hline
\end{tabular}

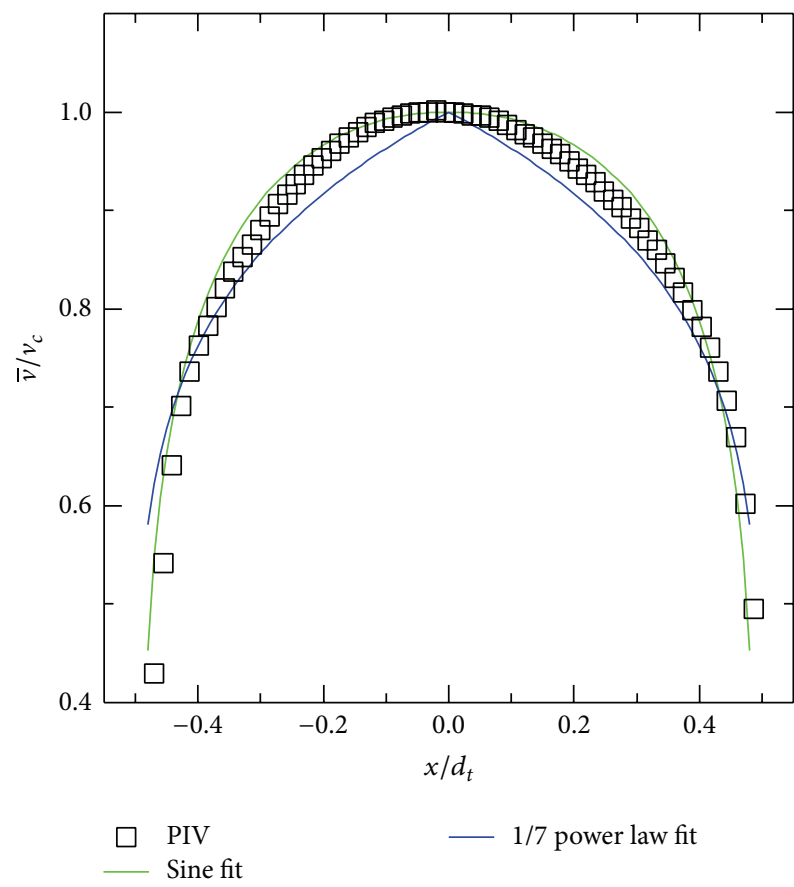

FIGURE 8: PIV data with a sine fit approach together with the classical $1 / 7$ power law at $y / d_{t}=0.097$ above the injection pipe exit.

this was a somewhat unexpected result. This holds true for the core of the jet, $0<x / d_{t}<0.35$, where our results are in close agreement with those reported in Eggels et al. [21] despite the lower Reynolds number for the pipe flow. In the outer part of the flow, $0.35<x / d_{t}<0.5$, the agreement becomes less prominent, but we find a good similarity with the results from den Toonder and Nieuwstadt [20]. This might be a Reynolds number effect; that is, the data from Eggels et al. [21] were recorded at $\operatorname{Re}_{j, b}=5400$ while those of den Toonder and Nieuwstadt [20] were taken at $\operatorname{Re}_{j, b}=17800$ which is much closer to our results. There seems to be no consensus in the literature, whether [29] or not [30] the magnitude and the position of the near wall peak of the stream wise $\left(v^{\prime}\right)$ turbulence intensity in a fully developed pipe flow is invariant with Reynolds number. Since this discussion is beyond the scope of this paper, we followed a pragmatic approach in choosing the experimental data according to references given in Table 2. The interested reader might find in both papers exhaustive references to pipe flow measurements and we conclude with a reasonable similarity between the present jet exit measurements and previous pipe flow results. Compared to the tube exit measurements reported in $[17,19,31]$, the measurements from our experiments have the same shape; however, the literature values fall consistently below our results (Figure 9(b)). Additionally, all experimental data miss the expected turbulence intensity increase in the mixing zone region between the jet-core and ambient, $0.45<x / d_{t}<0.5$. The latter issue might be caused by an insufficient spatial resolution such that the peak value is missed, but might not explain the lower magnitudes in the core of the jet. As argued above, it is expected that the flow past the tube exit keeps its memory to the pipe flow origin at least in the jet-core and we find also a considerable difference between the jet exit and the pipe flow data. Also the data presented might suffer from the digitization process and the subsequent renormalization, but the procedure used for the jet exit and the pipe flow data were similar.

Finally, the radial velocity fluctuations $u^{\prime} / v_{c}$ (Figure 9(c)) fit into the picture. Again we find a reasonable similarity between our and the pipe flow data in the jet core, while the results differ-as expected-close to the mixing zone. There was only one reference outlining past measurements of $u^{\prime} / v_{c}$ which is considerably below our measurement. At the moment, this subject must be left open for a refined analysis and future discussions. Overall, our measurements agree well with pipe flow results but show a lack of agreement with past tube exit measurements.

\section{In-Vessel Results}

For the helium-rich air layer build-up helium was injected for a certain amount of time determined in scoping tests through a tube $2 \mathrm{~m}$ below the vessel dome until the helium concentration of 0.37 molar fraction is reached. The initial density profile for the experiment measured at $y=-648 \mathrm{~mm}$ off-axis (Figure 1) are shown in Figure 10. In the lower part of the vessel we have initially an air atmosphere at room temperature $\left(T \approx 22^{\circ} \mathrm{C}, p=0.994\right.$ bar $)$ while the helium-air mixture with a lower density is trapped in the vessel dome.

The measurement is initiated by opening a valve to release the air-helium jet $\left(t_{0}=0 \mathrm{~s}\right)$ and the entire experiment is finished when the helium-rich layer is completely eroded such that we measure similar densities in the entire vessel $(t \simeq 5300 \mathrm{~s})$. Mean velocities and velocity fluctuations were measured using PIV in three regions (A to C; Figure 1) of the flow, all above, and around the axis of the injection pipe. These measurements have been processed to produce 


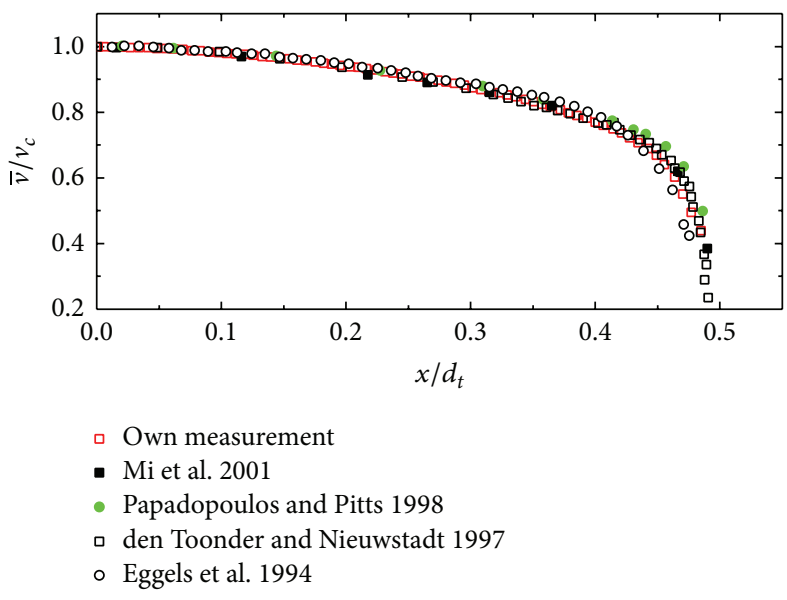

(a)

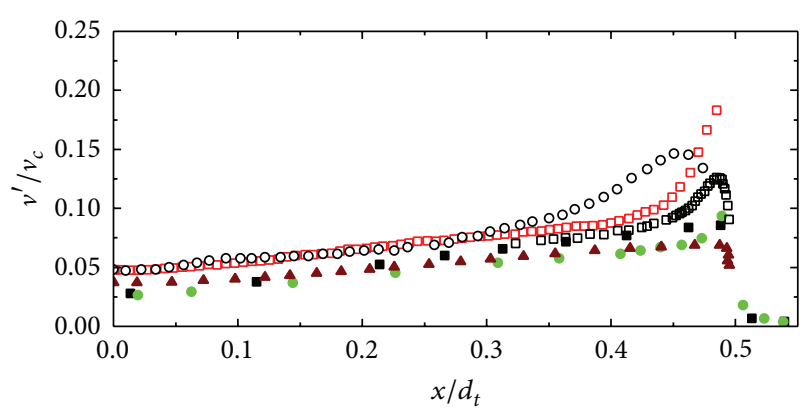

- Own measurement

- Mi et al. 2001

- Papadopoulos and Pitts 1998

- Boguslawski and Popiel 1979

- den Toonder and Nieuwstadt 1997

- Eggels et al. 1994

(b)

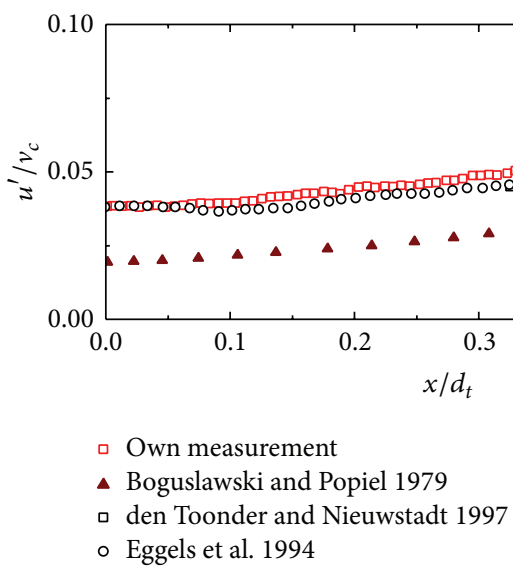

(c)

FIGURE 9: Comparison of mean and turbulence statistics $\left(\bar{v}, v^{\prime}, u^{\prime}\right)$ of own tube exit measurements with straight pipe tube exit measurements from the literature and turbulent pipe flow results.

averaged values over a time period of $204.8 \mathrm{~s}$. The measured data refer to the time $t_{c}$ in the middle of this data averaging period, that is, $\pm 102.4 \mathrm{~s}$ around each specified data item. An overview of the PIV recordings performed during the experiment is given in Table 3.

Velocity magnitude maps $\left(|\bar{v}|=\sqrt{u^{2}+v^{2}}\right)$ with the corresponding turbulent kinetic energy maps $k$, (11), measured in the air/helium jet impinging onto the helium-rich air layer from below are presented in Figure 11 for selected instances in time (Table 3). The time steps cover the instance right past the beginning of the injection (Figure 11(a)), later in time when the erosion process has proceeded (Figures 11(b) and 11(c)), and finally the time the erosion zone is going to leave the FOV above $y=6900 \mathrm{~mm}$ (Figure 11(d)). Streamlines have been calculated from the underlying velocity field to guide the eye. These streamlines are identical for corresponding $|\bar{v}|-k$ maps. The injection tube axis at $\approx 650 \mathrm{~mm}$ was marked with a dashed line. Since the seeding particles for the PIV measurements are transported with the jet, the helium layer unaffected by the jet is nonseeded; consequently, no velocities are recorded in those small areas; this becomes visible for the $k$ maps in the top parts of Figures $11(\mathrm{~g})$ and $11(\mathrm{~h})$ where we find a region with $k=0$.

Additionally, to assess the quality of the data, we have thresholded the statistical quantities being calculated from minimal 900 valid vectors which results in the abrupt changes of the quantities in areas with a lower number of valid vectors. One finds also minor horizontally and vertically oriented distortion zones which were caused by the instrumentation wires used for the temperature and mass spectrometer measurements (Figure 2). These areas were masked during the analysis and the resulting gaps were filled by linear interpolation from the surrounding quantities to facilitate the calculation of streamlines.

Due to its momentum, the jet (primary flow) continuously penetrates upwards into the helium-rich layer. Caused by the negative buoyancy, the axial velocity experiences a strong deceleration in the vicinity of the helium-rich layer 


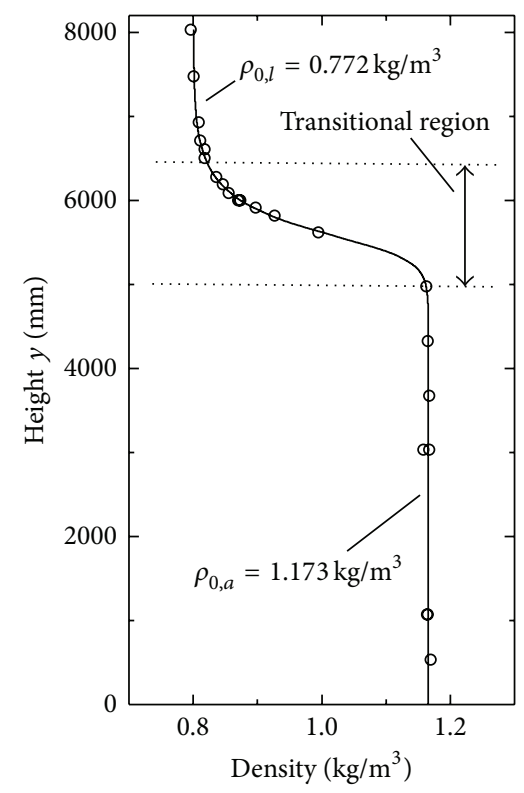

FIGURE 10: Initial gas density $\left(t_{0}=0 \mathrm{~s}\right)$ as a function of height in the vessel.

TABLE 3: Labels of the PIV measurements performed (N01 to N07), FOV (A to C), and central sampling time $t_{c}$.

\begin{tabular}{lcc}
\hline Number & Position & $t_{c}$ \\
\hline N01 & A & 111 \\
N02 & B & 715 \\
N03 & B & 978 \\
N04 & B & 1213 \\
N05 & C & 1795 \\
N06 & C & 2030 \\
N07 & C & 2286 \\
N08 & C & 2550 \\
\hline
\end{tabular}

(the mixing zone) (Figures 11(b) and $11(\mathrm{c})$ ), and the jet is finally stopped. Fluid accumulates in a continuous process in this mixing zone and part of the fluid consisting in an airhelium mixture is flowing back in a narrow annular region around the upward flowing jet as indicated by the streamlines (Figure 11(a)). Consequently, the jet decelerates additionally because the downwards annular flow slows down the upward jet flow and part of the annular flow is reentrained into the rising jet. The main difference between the early stage of the erosion process (N01) and a later time (N02, N05, and N08) is the stronger confinement of the flow around the jet (Figures 11(a) versus 11(b)); this difference can be attributed to three effects: firstly, the spreading through entrainment of ambient fluid in the jet with downstream distance, secondly the increasing resistance the helium-rich layer that imposes onto the jet propagation, and thirdly, the continuous entrainment and downwards transport of the helium-rich layer which decreases the "ambient" density. Initially (N01) the jet penetrates into the transitional part of the helium-rich layer, $5000<y<6000 \mathrm{~mm}$, where the density decreases from 1.166 to $0.80 \mathrm{~kg} / \mathrm{m}^{3}$ (Figure 10). Consequently, the negative buoyancy initially imposed by the layer onto the jet is weaker compared with later instances in time, when this transitional region is eroded (N02 $\rightarrow \mathrm{N} 05 \rightarrow \mathrm{N} 08$ ); see also [8]. Also initially (N01) the jet at the tube exit experiences a positive buoyancy due to the density difference between jet and ambient, $\Delta \rho_{0, j a} \approx 11 \%$, which partly compensates the velocity decay natural for a jet not impinging onto a helium-rich layer. This positive buoyancy decreases over time through the downwards transport of the helium-rich layer. At the start of the erosion process, the secondary flow structure is a downwards oriented annular type. During the erosion process, this structure changes to a horizontally oriented mushroom type of flow (Figure $11 \mathrm{~N} 02$ to N08) as indicated by the streamlines. This erosion process is shown from another perspective for the velocity field recorded at the fixed position $\mathrm{B}$ (Figure 12) at three different instances in time. The erosion front moves upwards ( $\mathrm{N} 02 \rightarrow \mathrm{N} 03 \rightarrow \mathrm{N} 04)$, the radius of curvature for the streamlines increases, and finally the mixing zone is above the FOV (N04).

The observations for the velocity magnitude field equally apply to the turbulent kinetic energy maps (Figures 11(e) versus $11(\mathrm{~g}))$. While we initially find $k \approx 0.1 \mathrm{~m}^{2} / \mathrm{s}^{2}$ in the core of the jet (N01), the kinetic energy is later distributed to a larger area such that we find $k \approx 0.03 \mathrm{~m}^{2} / \mathrm{s}^{2}$ (N08). In the zero mean velocity region (Figures $11(\mathrm{~b})$ and $11(\mathrm{f})$ - mark $\mathrm{A}$ ) we record significant turbulent kinetic energy indicating that the jet deceleration and redirection introduces turbulence in regions with zero mean velocity.

The three temperature contour maps presented in Figure 13 correspond to the PIV measurements in Figure 11 for times N01, N02, and N05 and have also been averaged over $\Delta t=204.8 \mathrm{~s}$. The temperature contour maps were calculated by linearly interpolating the temperatures between the thermocouple measurement locations depicted by black crosses. The jet was injected on purpose with a slightly elevated temperature compared with the ambient and the helium-rich layer to make temperature contour maps possible. It should be noted that the density difference between jet and ambient caused by $\Delta T=5^{\circ} \mathrm{C}$ is very small when compared with initial density difference of $11 \%$ (Table 1). For N01 right after the beginning of the injection of the jet, the somewhat colder $\left(T \approx 20^{\circ} \mathrm{C}\right.$ ) helium-rich layer becomes visible in the vessel dome.

The off-axis directed momentum of the jet results in a weak response of the helium layer such that the temperature map shows a left-right asymmetry which persists during the entire experiments. The helium downwards transport is depicted for time N02 by the almost uniform temperature region between the jet injection level and the helium-rich layer. Later in time (N05) part of the layer is already eroded.

The helium molar fraction $\chi_{\text {he }}$ in the jet axis $(x=$ $-648 \mathrm{~mm}$ ) as a function of time for some selected vertical locations is presented in Figure 14. Additionally, the molar fraction of helium measured at the tube exit in the jet is provided with the measurement at location $y=3000 \mathrm{~mm}$. Initially, the helium content of the jet decays from $\chi_{\mathrm{he}} \approx 0.13$ 


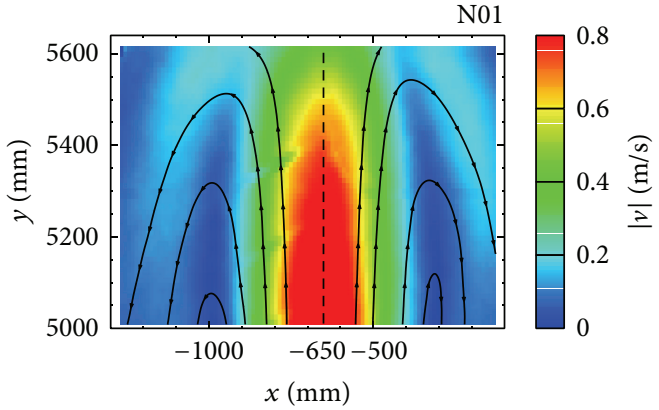

(a)

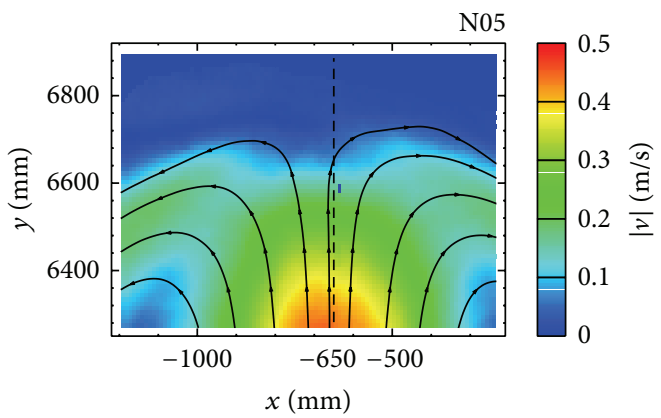

(c)

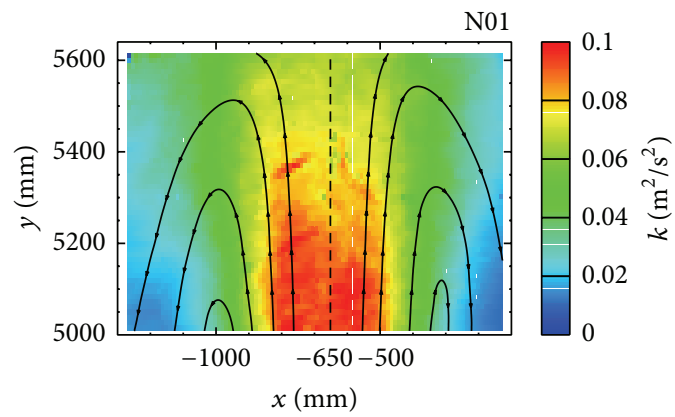

(e)

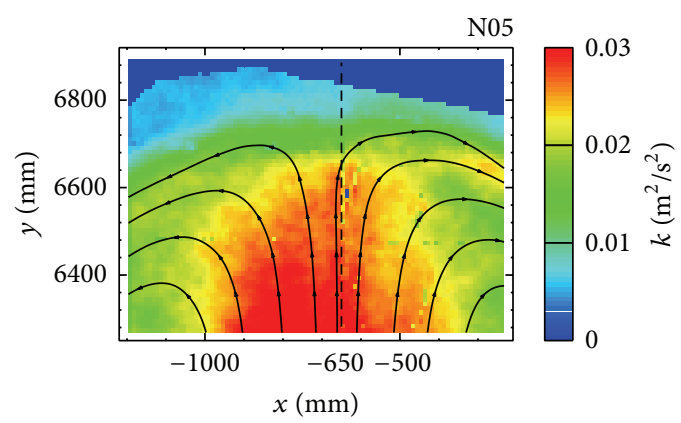

(g)

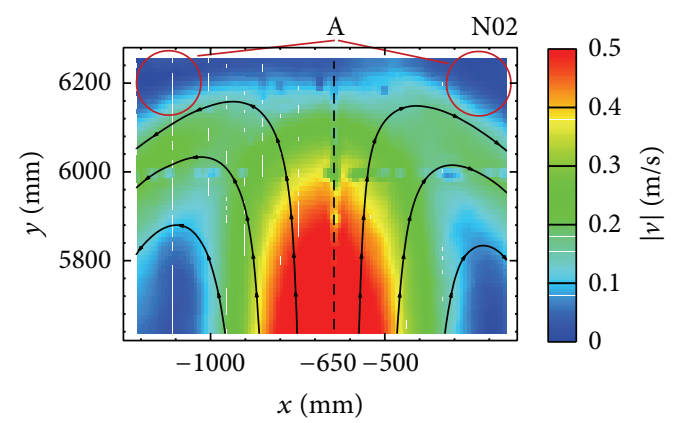

(b)

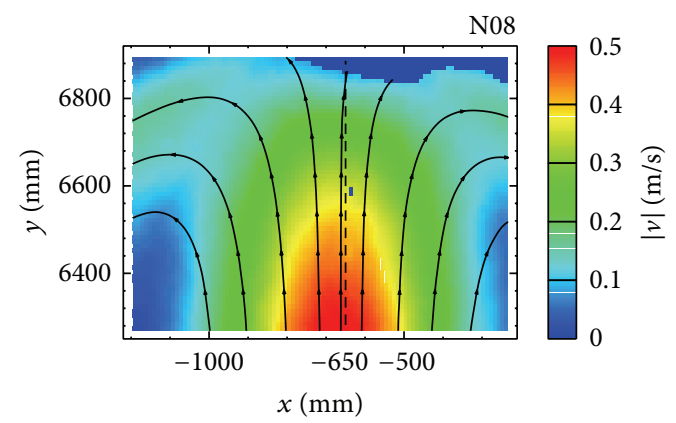

(d)

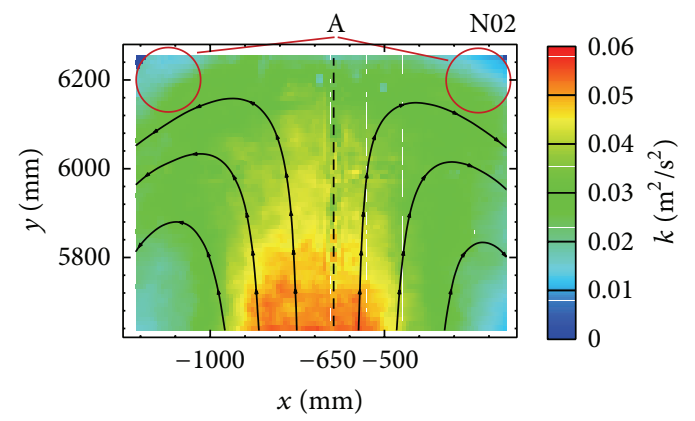

(f)

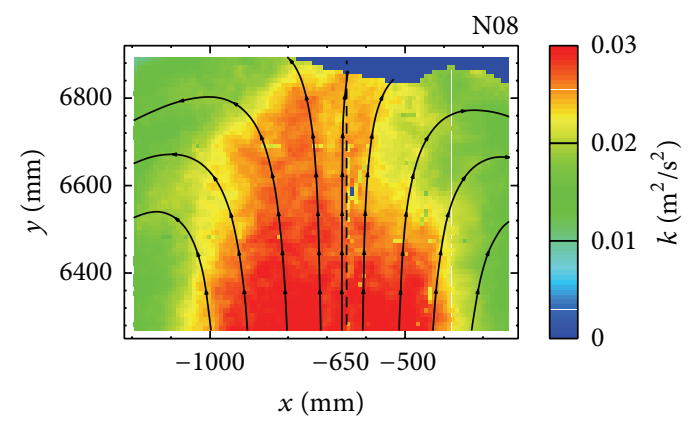

(h)

FIGURE 11: Selected velocity magnitude maps $|v|((\mathrm{a})$ to (d)) and the corresponding turbulent kinetic energy $k$ maps ((e) to (h)) recorded during the erosion process of the helium-rich layer.

at the tube exit $(y=3000 \mathrm{~mm})$ to $\chi_{\text {he }} \approx 0.05$ at position $y=$ $4326 \mathrm{~mm}$ considerably below the helium-rich layer through the entrainment of ambient gas from the environmentmostly pure air at this early stage. Caused by the continuous erosion of the helium-rich layer at the top and the downwards transport of helium, the helium content of the ambient increases such that $\chi_{\text {he }}$ in the jet at position $y=4326 \mathrm{~mm}$ increases accordingly over time. At the initial stage of the erosion process, it is expected that the secondary flow described previously is not passing all the way down to the jet orifice and 


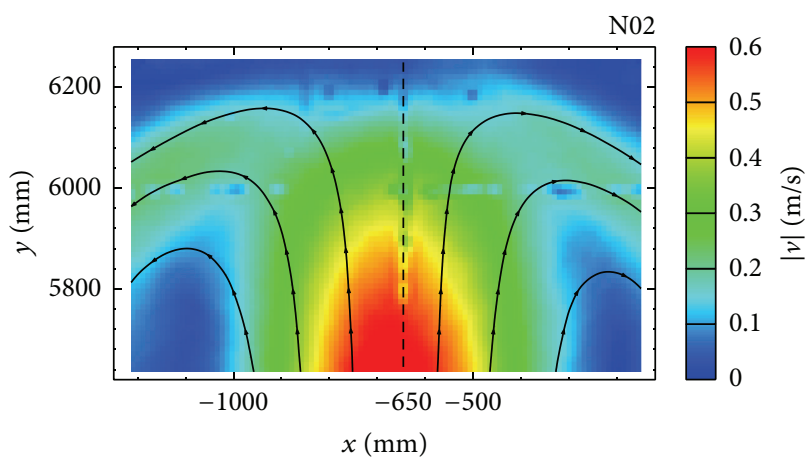

(a)

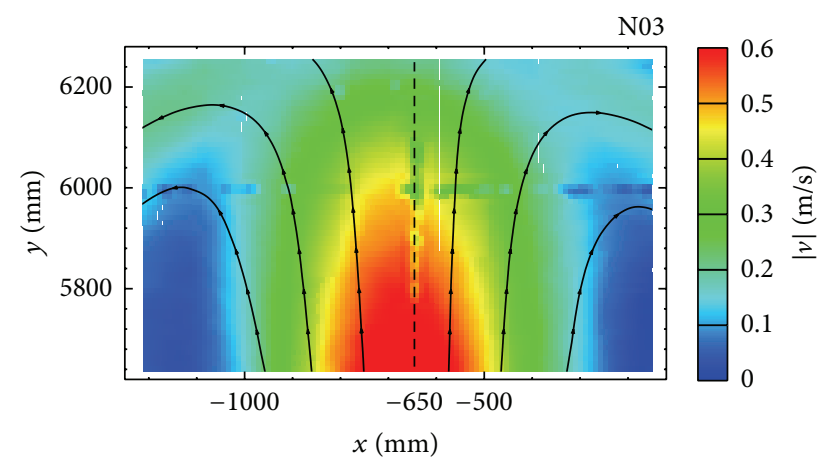

(b)

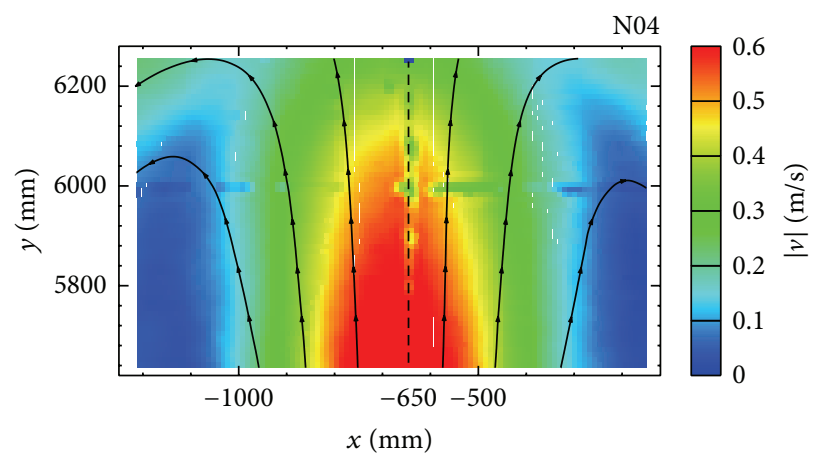

(c)

FIGURE 12: Development of the velocity magnitude field for position B during time steps $t=715,978$, and $1213 \mathrm{~s}$.
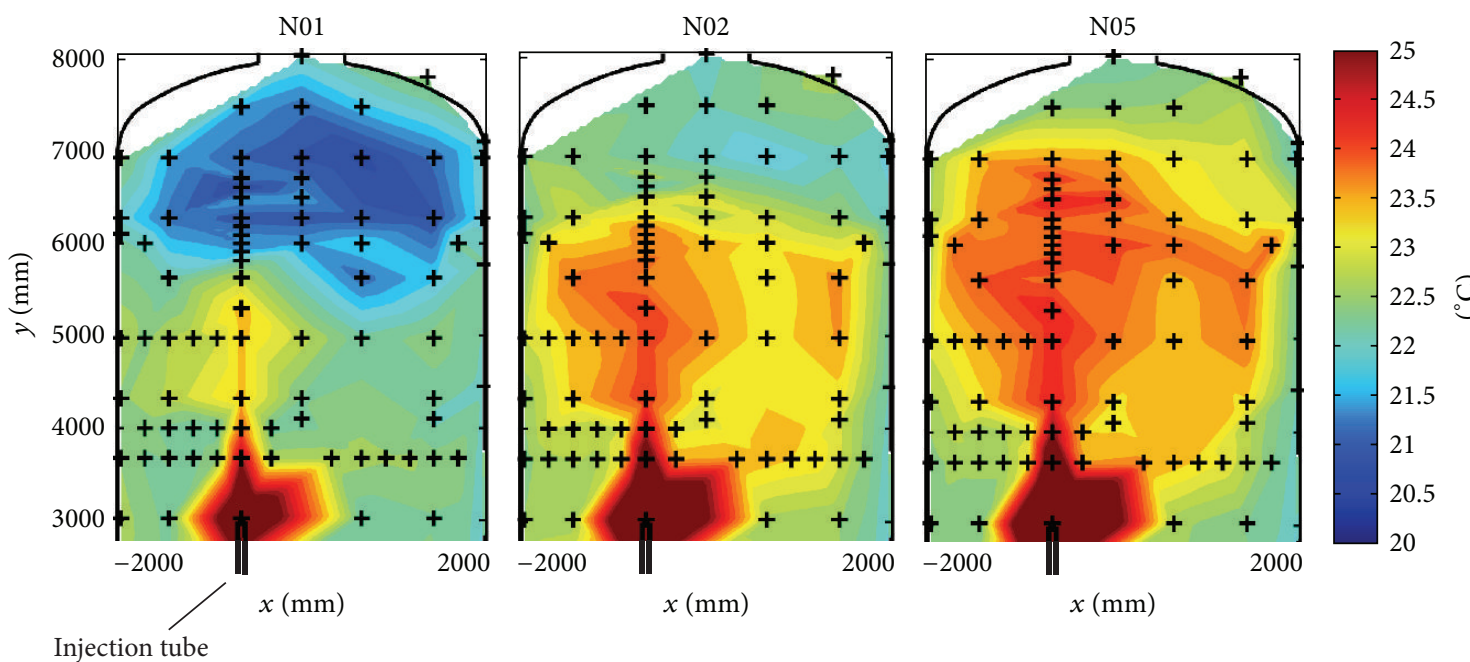

Figure 13: Temperature contour maps for the entire vessel above the injection level.

eventually even further down to the vessel bottom, but down to an intermediate level where the density of the annular flow equals the density of the surrounding such that the fluid starts to spread radially as described in $[8,32]$. Consequently, the measurement location at the bottom of the vessel $(y=$ $1076 \mathrm{~mm}$ ) indicates until $t \approx 1500 \mathrm{~s}$ no helium transport into this region. The helium content measured at points initially located in the helium-rich layer $(y \geq 6000 \mathrm{~mm}$ ) decays in time such that locations closer to the tube exit decay earlier, while locations closer to the vessel dome remain initially almost constant and unaffected by the jet but decay later in time. The helium content for all positions approaches finally a common level of $\chi_{\text {he }} \approx 0.15$; a state for which the gas in the entire vessel is homogeneously mixed. 

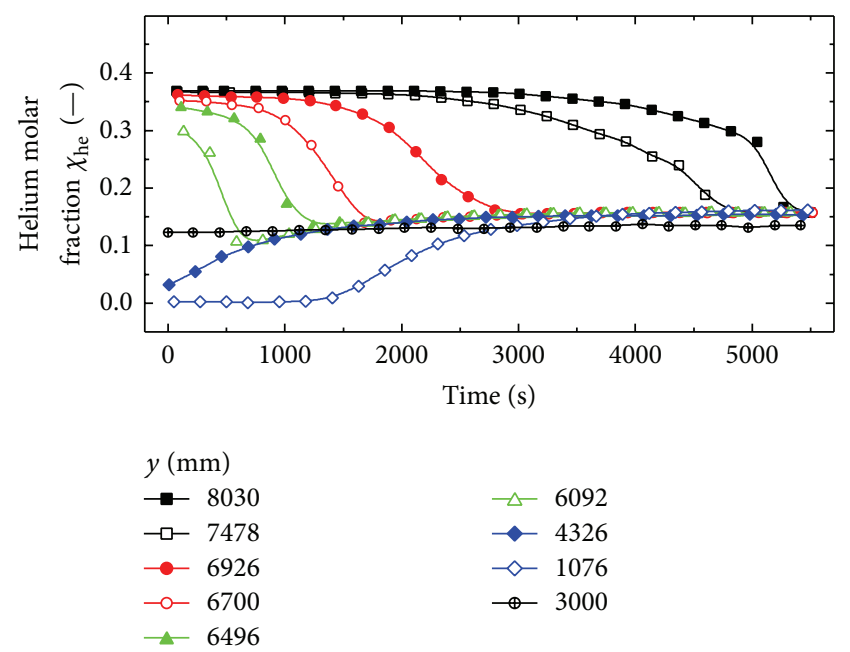

FIGURE 14: Helium molar fraction measured for selected locations in the jet axis ( $x=-650 \mathrm{~mm}$ ) as a function of time with the vertical measurement location as parameter. The molar fraction at the tube exit is provided at $y=3000 \mathrm{~mm}$.

\section{Conclusions}

Computational fluid dynamics (CFD) codes are increasingly used for safety analysis to simulate transient containment conditions after postulated severe accident scenarios in nuclear power plants (NPPs). Consequently, the reliability of such codes must be benchmarked against experimental data obtained preferentially in large scale facilities to limit scaling effects. Such an experiment was carried out in the large scale PANDA facility at the Paul Scherrer Institute in Switzerland for an OECD/NEA benchmark. The experiment highlights the interaction of a vertical air jet with a helium stratification.

For the tube exit measurements we find an excellent agreement between our results for the mean axial velocity and those quoted in the literature, irrespective of the experimental setup-pipe exit or turbulent pipe flow. And we have found a reasonable agreement between the shape of the classical $1 / 7$ power law and the experimental data, but a much better approximation is provided by the sine law introduced. Although the general shape of the measurements of the velocity fluctuations from the literature performed close to the tube exit is similar to our measurements, their magnitudes are systematically below our results. Additionally, all tube exit measurements miss the expected turbulence intensity increase in the mixing zone region between the jet-core and ambient. Somewhat unexpected we found a better agreement between our measurements and the pipe flow results for the velocity fluctuations compared with other jet exit measurements.

The vertical jet discharges initially into a positively buoyant environment and after a certain distance, the ambient density continuously decreases such that the positively buoyant jet becomes increasingly negatively buoyant when penetrating the helium-rich layer and the axial velocity decays very rapidly. Fluid accumulates in this mixing zone and a part of the fluid is flowing back in a small annulus around the upward flow. By this transient mechanism, the helium-rich layer is continuously eroded and helium is transported into lower parts of the test section such that the jet initial positive buoyancy decays over time.

\section{Conflict of Interests}

The authors declare that there is no conflict of interests regarding the publication of this paper.

\section{Acknowledgments}

The authors would like to thank the staff members Max Fehlmann and Simon Suter for their engaged support in conducting these experiments.

\section{References}

[1] W. D. Baines, "Entrainment by a plume or jet at a dens ity interface," Journal of Fluid Mechanics, vol. 68, no. 2, pp. 309320, 1975.

[2] C.-J. Chen and W. Rodi, "Vertical turbulent buoyant jets: a review of experimental data," NASA STI/Recon Technical Report A 80, 1980.

[3] E. J. List, “Turbulent jets and plumes," Annual Review of Fluid Mechanics, vol. 14, pp. 189-212, 1982.

[4] G. Lipari and P. K. Stansby, "Review of experimental data on incompressible turbulent round jets," Flow, Turbulence and Combustion, vol. 87, no. 1, pp. 79-114, 2011.

[5] C. G. Ball, H. Fellouah, and A. Pollard, "The flow field in turbulent round free jets," Progress in Aerospace Sciences, vol. 50, pp. 1-26, 2012.

[6] J. S. Turner, Buoyancy Effects in Fluids, Cambridge University Press, 1979.

[7] R. Kapulla, D. Paladino, G. Mignot, R. Zboray, and S. Gupta, "Break-up of gas stratification in LWR containment induced by negatively buoyant jets and plumes," in Proceedings of the 17th International Conference on Nuclear Engineering (ICONE '09), pp. 657-666, ASME, July 2009.

[8] R. Kapulla, G. Mignot, and D. Paladino, "Dynamics of helium stratifications eroded by vertical air jets with different momenta," in Proceedings of the 15th International Topical Meeting on Nuclear Reactor Thermalhydraulics, vol. 2009, pp. 657-666, ASME, 2013.

[9] G. Mignot, R. Kapulla, R. Zboray, N. Erkan, and D. Paladino, "Parametric study of containment gas stratification break-up by vertical fluid release," in Proceedings of the 13th International Topical Meeting on Nuclear Reactor Thermal Hydraulics, Paper NURETH13-1087, Kanazawa, Japan, September 2009.

[10] R. Zboray and D. Paladino, "Experiments on basic thermalhydraulic phenomena relevant for LWR containments: gas mixing and transport induced by buoyant jets in a multi-compartment geometry," Nuclear Engineering and Design, vol. 240, no. 10, pp. 3158-3169, 2010.

[11] G. Yadigaroglu, M. Andreani, J. Dreier, and P. Coddington, "Trends and needs in experimentation and numerical simulation for LWR safety," Nuclear Engineering and Design, vol. 221, no. 1-3, pp. 205-223, 2003.

[12] D. Paladino, R. Zboray, P. Benz, and M. Andreani, "Three-gas mixture plume inducing mixing and stratification in a multicompartment containment," Nuclear Engineering and Design, vol. 240 , no. 2, pp. 210-220, 2010. 
[13] M. Houkema, N. B. Siccama, J. A. Lycklama à Nijeholt, and E. M. J. Komen, "Validation of the CFX4 CFD code for containment thermal-hydraulics," Nuclear Engineering and Design, vol. 238, no. 3, pp. 590-599, 2008.

[14] A. Epiney, K. Mikityuk, and R. Chawla, "Heavy-gas injection in the generation IV gas-cooled fast reactor for improved decay heat removal under depressurized conditions," Nuclear Engineering and Design, vol. 240, no. 10, pp. 3115-3125, 2010.

[15] M. Andreani, K. Haller, M. Heitsch et al., "A benchmark exercise on the use of CFD codes for containment issues using best practice guidelines: a computational challenge," Nuclear Engineering and Design, vol. 238, no. 3, pp. 502-513, 2008.

[16] A. Dewan, Tackling Turbulent Flows in Engineering, Springer, 2011.

[17] J. Mi, D. S. Nobes, and G. J. Nathan, "Influence of jet exit conditions on the passive scalar field of an axisymmetric free jet," Journal of Fluid Mechanics, vol. 432, pp. 91-125, 2001.

[18] G. Papadopoulos and W. M. Pitts, "Scaling the near-field centerline mixing behavior of axisymmetric turbulent jets," AIAA Journal, vol. 36, no. 9, pp. 1635-1642, 1998.

[19] L. Boguslawski and C. O. Popiel, "Flow structure of the free round turbulent jet in the initial region," Journal of Fluid Mechanics, vol. 90, no. 3, pp. 531-539, 1979.

[20] J. M. J. den Toonder and F. T. M. Nieuwstadt, "Reynolds number effects in a turbulent pipe flow for low to moderate Re," Physics of Fluids, vol. 9, no. 11, pp. 3398-3409, 1997.

[21] J. G. M. Eggels, F. Unger, M. H. Weiss et al., "Fully developed turbulent pipe flow: a comparison between direct numerical simulation and experiment," Journal of Fluid Mechanics, vol. 268, pp. 175-209, 1994.

[22] OECD-NEA, OECD-NEA-PSI CFD Benchmark Specification, National Environment Agency, 2013.

[23] E. Lemmon, M. Huber, and M. McLinden, NIST Standard Reference Database 23: Reference Fluid Thermodynamic and Transport Properties-REFPROP, Version 9.0, National Institute of Standards and Technology, Standard Reference Data Program, Gaithersburg, Md, USA, 2010.

[24] M. Raffel, C. Willert, S. Wereley, and J. Kompenhans, Particle Image Velocimetry, A Pratical Guide, Springer, Berlin, Germany, 2007.

[25] H. Albrecht, M. Borys, N. Damaschke, and C. Tropea, Laser Doppler and Phase Doppler Measurement Techniques, Springer, New York, NY, USA, 2003.

[26] L. J. De Chant, "The venerable 1/7th power law turbulent velocity profile: a classical nonlinear boundary value problem solution and its relationship to stochastic processes," Applied Mathematics and Computation, vol. 161, no. 2, pp. 463-474, 2005.

[27] J. Bendat and A. Piersol, Analysis and Measurement Procedures, John Wiley \& Sons, New York, NY, USA, 1986.

[28] G. Xu and R. Antonia, "Effect of different initial conditions on a turbulent round free jet," Experiments in Fluids, vol. 33, no. 5, pp. 677-683, 2002.

[29] M. Hultmark, S. C. C. Bailey, and A. J. Smits, "Scaling of nearwall turbulence in pipe flow," Journal of Fluid Mechanics, vol. 649, pp. 103-113, 2010.

[30] H. C. H. Ng, J. P. Monty, N. Hutchins, M. S. Chong, and I. Marusic, "Comparison of turbulent channel and pipe flows with varying Reynolds number," Experiments in Fluids, vol. 51, no. 5, pp. 1261-1281, 2011.
[31] G. Papadopoulos and W. M. Pitts, "A generic centerline velocity decay curve for initially turbulent axisymmetric jets," Journal of Fluids Engineering, vol. 121, no. 1, pp. 80-85, 1999.

[32] L. J. Bloomfield and R. C. Kerr, "Turbulent fountains in a stratified fluid," Journal of Fluid Mechanics, vol. 358, pp. 335356, 1998. 


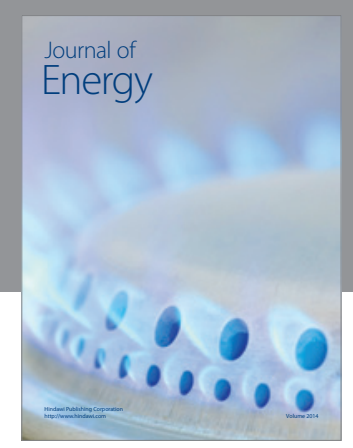

Journal of

Industrial Engineering
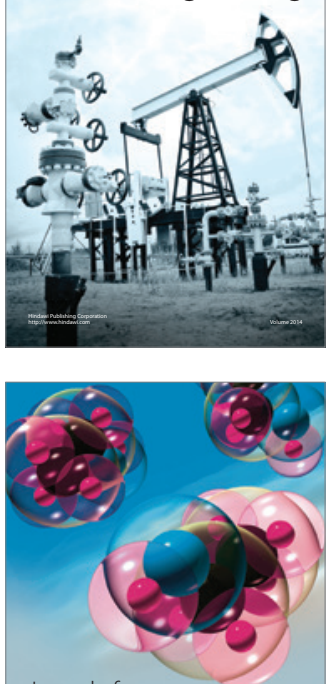

Fuels
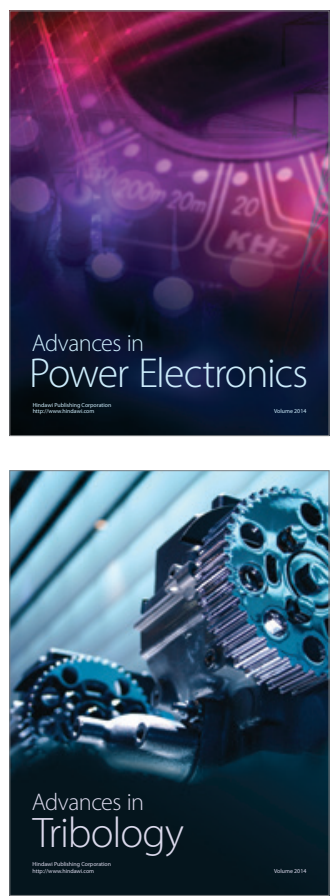

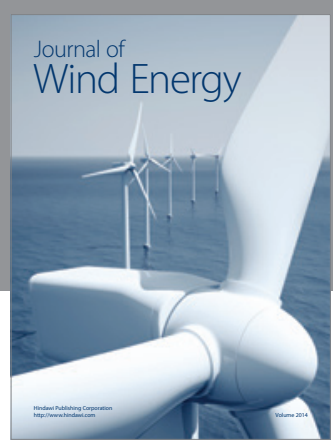

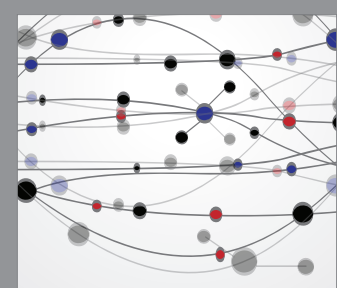

The Scientific World Journal

Submit your manuscripts at http://www.hindawi.com

Journal of

Structures
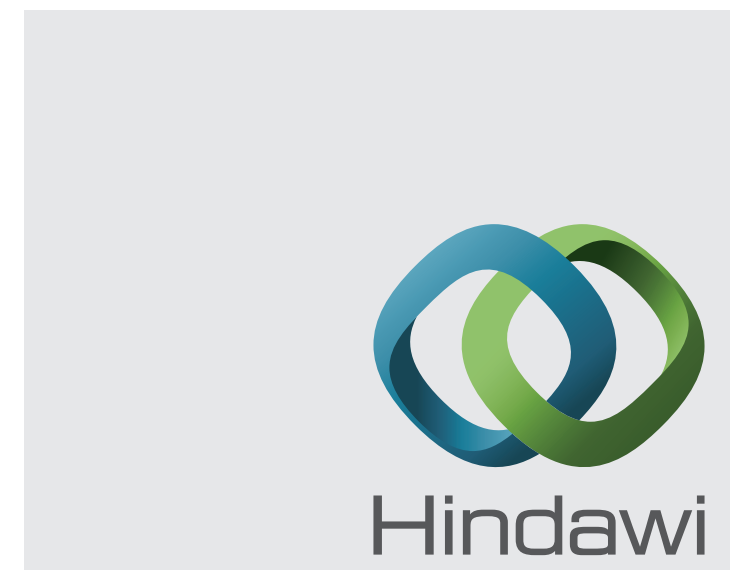

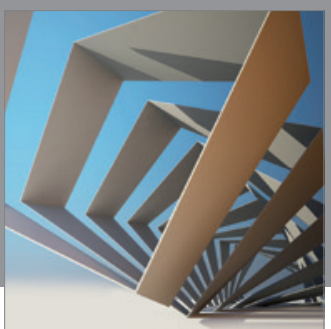

Rotating

Machinery
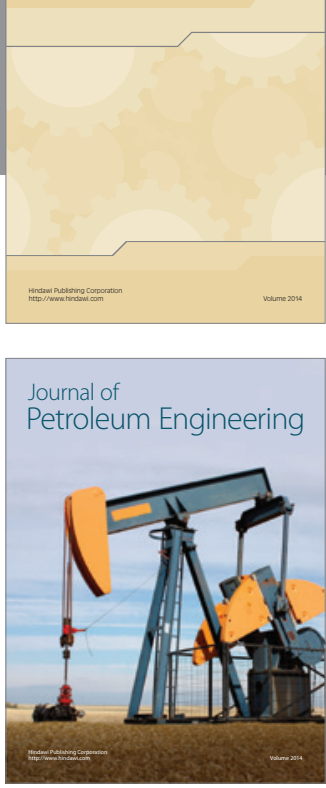

Journal of

Solar Energy
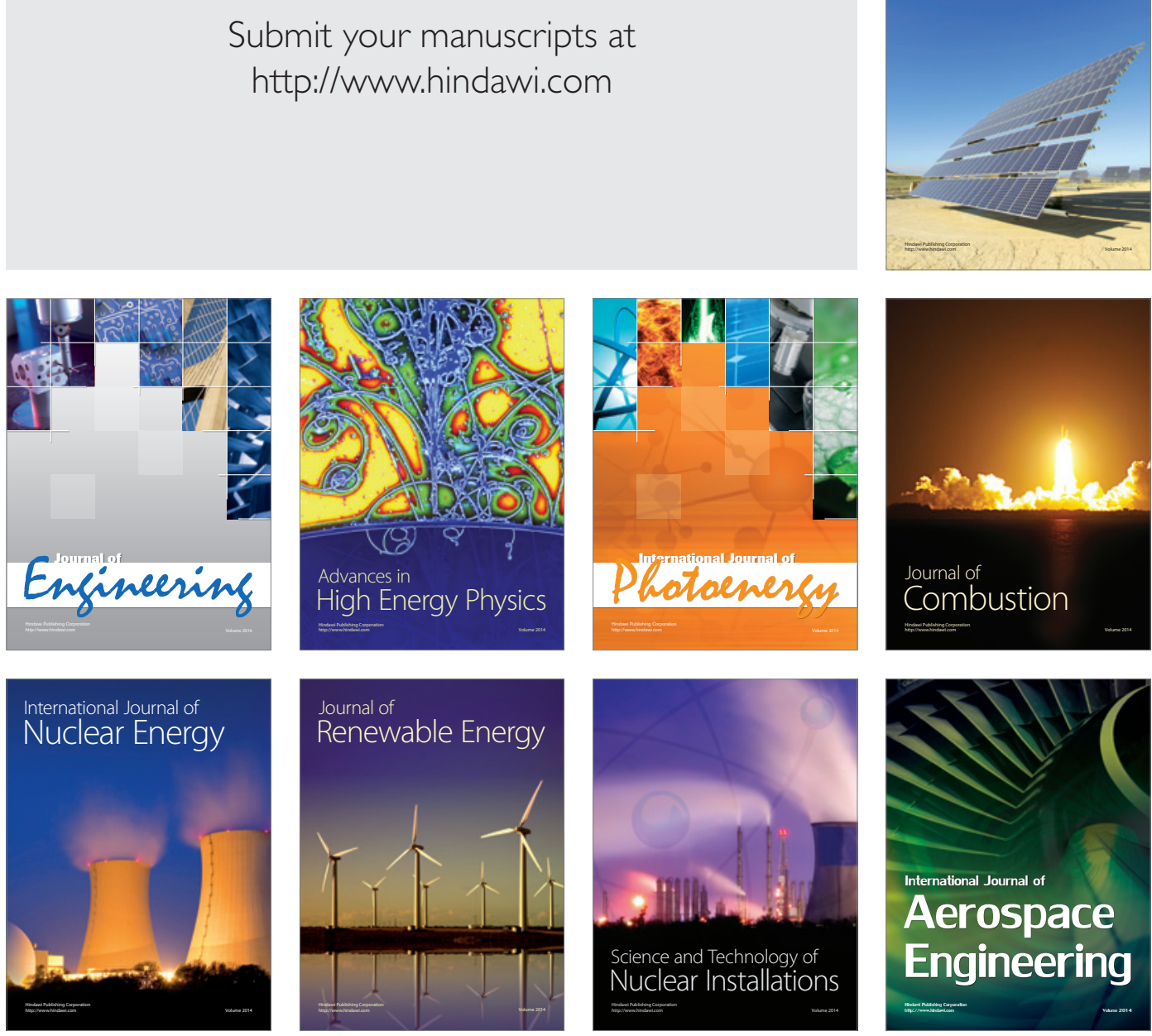